\title{
New hybrids of tacrine and indomethacin as multifunctional acetylcholinesterase inhibitors
}

\author{
Kamil Zawada ${ }^{1} \cdot$ Kamila Czarnecka $^{1} \cdot$ Małgorzata Girek $^{2} \cdot$ Paweł Kręcisz $^{1} \cdot$ František Trejtnar $^{3}$ - Jana Mandíková ${ }^{3}$. \\ Jakub Jończyk ${ }^{4} \cdot$ Marek Bajda $^{4} \cdot$ Mariusz Staśkiewicz ${ }^{5} \cdot$ Przemysław Wójtowicz $^{1} \cdot$ Katarzyna Dziubek $^{6}$. \\ Robert Skibiński ${ }^{7} \cdot$ Paweł Szymański $^{1}$ (i)
}

Received: 14 January 2020 / Accepted: 22 July 2020 / Published online: 29 July 2020

(c) The Author(s) 2020

\begin{abstract}
A new series of hybrid compounds were designed, consisting of anti-AChE and BuChE activity components with an antiinflammatory component. A series of 9-amino-1,2,3,4-tetrahydroacridine and indomethacin derivatives were synthesized. All compounds were created using alkyldiamine with different chain lengths as a linker. Various biological activities were evaluated, including inhibitory activity against $\mathrm{AChE}$ and $\mathrm{BuChE}$. The tested compounds showed high inhibitory activities against cholinesterases. The $\mathrm{IC}_{50}$ values for all compounds ranging from $10 \mathrm{nM}$ to $7 \mu \mathrm{M}$. The potency of inhibition was much higher than well-known AChE and BuChE inhibitors (tacrine and donepezil). Compound $\mathbf{3 h}$ had the strongest inhibitory activity; kinetic studies showed it to have a mixed-type of acetylcholinesterase inhibition properties. The cytotoxicity of the newly-synthesized compounds against HepG2 (hepatocarcinoma cells) and EA.hy96 (human vein endothelial cells) cell lines was determined using the MTT and MTS tests. All investigated compounds presented similar cytotoxic activity against HepG2 and EA.hy926 cell line, ranged in micromolar values. Compounds with longer linkers showed higher antioxidant activity. The most active compound was $\mathbf{3 h}$. Docking studies confirmed interactions with important regions of AChE and BuChE. Its multifunctional properties, i.e. high activity against $\mathrm{AChE}$ and $\mathrm{BuChE}$, antioxidant activity and low cytotoxicity, highlight $\mathbf{3 h}$ as a promising agent for the treatment of $\mathrm{AD}$.
\end{abstract}

Keywords Acetylcholinesterase inhibitors · Alzheimer's disease $\cdot$ Indomethacin $\cdot$ Multifunctional drugs

$\begin{array}{ll}\text { Abbreviations } \\ \mathrm{AD} & \text { Alzheimer's disease } \\ \mathrm{AChE} & \text { Acetylcholinesterase } \\ \mathrm{BuChE} & \text { Butyrylcholinesterase } \\ \mathrm{ACh} & \text { Acetylcholine } \\ \mathrm{A} \beta & \beta \text {-Amyloid }\end{array}$

Electronic supplementary material The online version of this article (https://doi.org/10.1007/s11696-020-01295-y) contains supplementary material, which is available to authorized users.

Paweł Szymański

pawel.szymanski@umed.lodz.pl

1 Department of Pharmaceutical Chemistry, Drug Analysis and Radiopharmacy, Faculty of Pharmacy, Medical University of Lodz, Muszynskiego 1, 90-151 Lodz, Poland

2 Animal House, Faculty of Pharmacy, Medical University of Lodz, Muszynskiego 1, 90-151 Lodz, Poland

3 Department of Pharmacology and Toxicology, Faculty of Pharmacy in Hradec Kralove, Charles University, Heyrovskeho 1203, 50005 Hradec Králové, Czech Republic
ATCh Acetyltiocholine

CNS Central nervous system

MAPT Microtubule-associated protein tau

NFTs Neurofibrillary tangles

NSAIDs Nonsteroidal anti-inflammatory drugs

PGH2 Prostaglandin $\mathrm{H} 2$

CDMT 2-Chloro-4,6-dimethoxy-1,3,5-triazine

THF Tetrahydrofuran

4 Department of Physicochemical Drug Analysis, Faculty of Pharmacy, Jagiellonian University Medical College, Medyczna 9, 30-688 Kraków, Poland

5 Adamed Group, Technical Development Department, Piłsudskiego 5, 95-200 Pabianice, Poland

6 International Centre for Cancer Vaccine Science, University of Gdansk, Wita Stwosza 63, 80-308 Gdańsk, Poland

7 Department of Medicinal Chemistry, Faculty of Pharmacy, Medical University of Lublin, Jaczewskiego 4, 20-090 Lublin, Poland 
ABTS Diammonium 2,2'-azino-bis(3-ethylbenzothiazoline-6-sulfonate)

DPPH 2,2-diphenyl-1-picrylhydrazyl

\section{Introduction}

The most common human neurodegenerative disease is Alzheimer's disease (AD) — which was described for the first time by Alois Alzheimer over 100 years ago. AD causes

a significant decrease in cognitive abilities in people over 60 years old (Obulesu and Jhansilakshmi 2014). The incidence of AD increases with age: rising from 5\% among people aged $65-50 \%$ over the age 85 (Townsend and Praticò 2005). The disease has a progressive and multifunctional character. Its development involves a lot of factors but a complete etiology has not been discovered (Tayeb et al. 2012) and no method exists which can be used to treat AD (Côté et al. 2012). Existing drugs only slow the progression of the disease and slightly improve patient comfort and everyday life. Previous studies of AD pathophysiology have examined the formation of $\beta$-amyloid deposits, prolonged oxidative stress, cholinergic system dysfunctions (Tayeb et al. 2012; Zimmer et al. 2014; Blass 2002), inflammations, aging, heart disease, hypertension, hypercholesterolemia, diabetes, gene mutations associated with the incorrect tau protein (Cataldo et al. 2010), as well as the imbalance of bio-metals (Tayeb et al. 2012; Huang et al. 2014).

Autopsy of AD patient shows cholinergic system dysfunction in the brain, as well as disorders of $\mathrm{ACh}$, choline, and choline acetyltransferase levels. AD is characterized by ongoing neuron damage, resulting in decreasing levels of neurotransmitters, mainly acetylcholine (ACh). This damage leads to a deterioration of cognitive abilities and memory loss (Zemek et al. 2014; Allgaier and Allgaier 2014; Volpato and Holzgrabe 2018).

Most of the currently used treatment methods are based on raising the ACh levels in synapses. Acetylcholinesterase $(\mathrm{AChE})$ and butyrylcholinesterase $(\mathrm{BuChE})$ facilitate the hydrolysis of acetylcholine in the synaptic cleft to choline and acetate. AChE is present in the Central Nervous System (CNS), neuromuscular synapses and red blood cells; BuChE in the CNS, liver, plasma and lungs. AChE hydrolyzes acetylcholine faster than BuChE (Czarnecka et al. 2017). Among the receptors of the cholinergic system, the most important are muscarinic (mAChRs) and nicotinic (nAChRs) acid. The former binds with G-protein, while the latter regulates the opening of ion channels. The M1 receptor subtype is present in large numbers in the hippocampus and cerebral cortex and plays an important role in cognitive abilities and learning.
M1 muscarinic receptors can be a potential target for new medicines against AD. Some of the tested compounds acting on $\mathrm{M} 1$ receptors were found to reduce oxidative stress and amyloid $\beta$ deposits. The APP metabolic pathway is converted to nonamyloidogenic pathway when these compounds bind to the M1 receptors. Nicotinic receptors are transmembrane proteins that act as ionic channels. They consist of two subunits $(\alpha$ and $\beta$ ) and a hydrophilic centre. Nicotinic receptors mediate the flow of $\mathrm{Na}^{+}, \mathrm{K}^{+}$and $\mathrm{Ca}^{2+}$. Different kinds of nicotinic receptors with different combinations of $\alpha$ and $\beta$ subunits have been distinguished. Amyloid $\beta$ stimulates nicotinic receptors: nAChRs $\alpha 7$ at physiological concentrations, and $\alpha 7$ and $\alpha 4 \beta 2$ at pathological concentrations (Chen et al. 2013a, b; Volpato and Holzgrabe 2018).

Donepezil, rivastigmine and galantamine have been generally approved and are currently used in AD therapy. These compounds have the ability to cross the blood-brain barrier and inhibit AChE and BuChE (Chen et al. 2013a, b). Galantamine also has inhibitory properties against $\beta$-amyloid aggregation ( $\mathrm{Ng}$ et al. 2015). Memantine protects neurons against neurotoxic long-term overexposure of the glutamate receptor (Kurz and Perneczky 2011). Tacrine (9-amine1,2,3,4-tetrahydroacridine) has been withdrawn from use because of high its hepatotoxicity associated with increased serum alanine aminotransferase activity (Tayeb et al. 2012; Park et al. 2015).

Multitarget compounds such as hybrids composed of ligands with different pharmacological properties may represent an effective treatment for the complex pathophysiology of AD. New hybrids can act simultaneously on proteins involved in different metabolic pathways, such as AChE and cholinergic receptors. Another strategy is to create a hybrid acting on one protein but in different places: orthosteric inside the structure and allosteric on the surface. Ligands can modify the activity of the enzyme by changing its conformation. Hybrids may act on the G-protein associated with mAChRs receptors (Volpato and Holzgrabe 2018). Due to its strong inhibitory activity, tacrine derivatives connected with other moieties possess lower toxic properties and higher potency against AChE and BuChE (Chen et al. 2013a, b).

Tacrine hybrids have been investigated for over 20 years as a part of multi-target strategy of AD treatment. The desired hybrid is a molecule that can act simultaneously on different proteins, on different fragments of the same protein or on different pathways in the pathogenesis of the disease (Volpato and Holzgrabe 2018). Such compounds are 1,2,3,4-tetrahydroacridine and various 4-fluorobenzoic acid derivatives which demonstrate high potency against AChE, which selectivity was determined enzymatically (Szymanski et al. 2011). Similarly, tacrine hybrids with 2or 3-fluorobenzoic acid inhibit $\mathrm{AChE} / \mathrm{BuChE}$ and demonstrate inhibitory properties against amyloid- $\beta$ aggregation (Czarnecka et al. 2017). Tacrine-flavonoid hybrids show 
inhibitory potency against cholinesterases and amyloid- $\beta$ aggregation, and have metal chelating properties ( $\mathrm{Li}$ et al. 2013). Tacrine-flurbiprofen derivatives, characterized by higher inhibitory potency against $\mathrm{AChE}$ and BuChE than tacrine alone, inhibit amyloid- $\beta$ aggregation. They bind to the catalytic active site and peripheral anionic site of cholinesterase (Chen et al. 2013a, b). Tacrine hybrids with 8-hydroxycholine have neuroprotective, cholinergic, antioxidant and copper-complexing properties (FernandezBachiller et al. 2010).

The $\mathrm{A} \beta$ peptide is formed in the hydrolysis process from amyloid precursor protein (APP). $\beta$-Amyloid contains from 39 to 42 amino acids in the peptide chain (Meraz-Ríos et al. 2013). The most important in AD progression is $A \beta$, constructed from 40 amino acids (A $\beta 40)$ and 42 amino acids (Aß42) (Chen et al. 2013a, 2013b; Carreras et al. 2013). $\mathrm{A} \beta 42$ has higher potency to aggregate, be deposited in the CNS and is more neurotoxic than A $\beta 40$ (Czarnecka et al. 2017). They are created in the proteolysis of APP by two enzymes: $\beta$-secretase and $\gamma$-secretase (Tayeb et al. 2012).

Some gene mutations increase $A \beta$ production. Presenilins 1 and 2 are catalytic components of $\gamma$-secretase-mutations in presenilin genes disturb the $\mathrm{A} \beta 40 / \mathrm{A} \beta 42$ ratio (Townsend and Praticò 2005). High $\gamma$-secretase activity is also induced by cytokines and reactive oxygen species ( $\mathrm{Ng}$ et al. 2015). Additional $\beta$-amyloid binds with AChE in complexes, and these have much higher potency to create deposits than $\beta$-amyloid alone. Moreover, ions of zinc, copper and iron are catalysts in the process of forming $A \beta$ fibrils (Kurz and Perneczky 2011).

Other factors responsible for the development of $\mathrm{AD}$ is the synthesis of pathological MAPT (microtubule associated with protein tau), caused by gene mutations or an imbalance between kinases and phosphatases (Bajda et al. 2015; Medina and Avila 2014). Tau protein forms microtubules in the cell skeleton, particularly in the neurons. As a result of tau protein hyperphosphorylation, neurotoxic neurofibrillary tangles (NFT) are created (Obulesu and Jhansilakshmi 2014; McGeer 2000). The presence of A $\beta 42$ induces phosphorylation of tau protein (Tayeb et al. 2012; Kurz and Perneczky 2011; Berk et al., 2013). Pathological tau protein deposits result in the destabilization of the cytoskeleton and impaired conduction of impulses by axons (Bajda et al. 2015).

$\mathrm{AD}$ is characterized by the occurrence of inflammation in the brain (Townsend and Praticò 2005; Results of a follow-up study to the randomized Alzheimer's Disease Antiinflammatory Prevention Trial (ADAPT) 2013). Cytokines and prostaglandins play key roles in the complicated inflammatory process. Cytokines and prostaglandins are formed as a result of the action of cyclooxygenase (COX) (Townsend and Praticò 2005; McGeer 2000). Research showed that cytokines and prostaglandins ( $\mathrm{PGH}_{2}$ mainly) increase the synthesis of neurotoxic $\mathrm{A} \beta 42$ and its deposition in the CNS
(Results of a follow-up study to the randomized Alzheimer's Disease Anti-inflammatory Prevention Trial (ADAPT) 2013). The discovery that inflammation in the CNS is one of the main factors in $\mathrm{AD}$ development encouraged research into nonsteroidal anti-inflammatory drugs (NSAIDs) as potential treatments (Imbimbo et al. 2010). NSAIDs reduce the synthesis of prostaglandins and cytokines by inhibiting cyclooxygenase and secretase- $\gamma$ activity (Chen et al. 2013a, b; Cudaback et al. 2014; Prati et al. 2013). They exhibit good potency to decrease $\beta$-amyloid synthesis and create deposits. Research showed that the application of NSAIDs such as ibuprofen, flurbiprofen, sulindac and indomethacin can decrease of the synthesis of A $\beta 42$ and change the APP metabolism towards the less neurotoxic A $\beta 40$ (Prati et al. 2013).

Prolonged oxidative stress, i.e. an imbalance between the formation of oxygen and nitrogen free radicals and their removal, damages neurons. Free radicals are molecules that contain unpaired electrons. Oxidative stress damages DNA, lipids, carbohydrates and proteins inside the cells, and neurons are particularly vulnerable. Homoeostasis is maintained by endogenous antioxidants; however, in the case of prolonged imbalance, the cellular metabolism undergoes deregulation: phosphorylation intensifies, pro-inflammatory factors are formed and inflammation develops. $\mathrm{Cu}$ and $\mathrm{Fe}$ demonstrate strong redox properties and cause the formation of free oxygen radicals. Reactive forms of oxygen and nitrogen, together with pro-inflammatory factors, intensify the synthesis and aggregation of A $\beta$ 1-42 (Berk et al. 2013; Chen et al. 2011; Solleiro-Villavicencio and Rivas-Arancibia 2018).

In 2019, new hybrids of indole-3-acetic acid (IAA) and tacrine, connected by aliphatic linker were synthesized. The compound with six $\mathrm{C}$ atoms in the linker showed similar activity against $\mathrm{AChE}$ and higher activity against $\mathrm{BuChE}$ compared to tacrine. The most active compound was found to interact with both CAS and PAS of ChE. The basic nitrogen atom in the structure of the tested compound is responsible for binding with aromatic residues of amino acids in the active site of the enzyme. Additional de novo synthesized compounds do not show cytotoxic side effect relative to HepG2 (Cheng et al. 2019).

Research into tacrine-indomethacin hybrids and their potency to inhibit AChE and BuChE is ongoing. New hybrids based on acetylcholinesterase inhibitors have been found to both increase the ACh level and decrease the amount of $\beta$-amyloid deposits (Czarnecka et al. 2017). The aim of the present study was to create a new tacrine derivative with indomethacin moieties. It has previously been found that indomethacin with an indole ring in its structure with the $\mathrm{N}$ atom enhances the interaction between the hybrids and the cholinesterase in CAS and PAS (Cheng et al. 2019). Their potency against AChE and BuChE and 
selectivity was studied. Among the compounds obtained, those that showed the highest inhibitory potency were selected for cytotoxic and antioxidant evaluation. Moreover, molecular modeling and docking studies were performed to determine the structure of the synthesized compounds and how they interact with cholinesterases.

\section{Experimental: materials and methods}

Anhydrous $\mathrm{Na}_{2} \mathrm{SO}_{4}$ (Sigma-Aldrich) was used to dry the organic solutions. The solvents were then evaporated under vacuum with a rotary evaporator. Reactions were checked by TLC analysis, for which the 25 DC-Alufolien Kieselgel 60F254 (Merck) and UV Lamp (254 nm) were used. The reaction products were purified by flash chromatography (column $50 \mu \mathrm{m}$ SiHP, $12 \mathrm{~g}$ Interchim) with methylene chloride (methylene chloride: methanol: $30 \% \mathrm{NH}_{3} ; 10: 1: 0.2$ ) gradient elution. Melting points for $\mathbf{2 a}-\mathbf{2 h}$ and $\mathbf{3 a}-\mathbf{3 h}$ were measured in open capillaries on the electrothermal apparatus. The IR spectra of $\mathbf{2 a - 2 h}, \mathbf{3 a}-\mathbf{3 h}$ were recorded in attenuated total reflectance (ATR) mode (Thermo Scientific Nicolet 6700). Fourier transform infrared spectrometer with smart ITR diamond adapter (Madison, Wisconsin USA) was used. An Agilent Accurate-Mass Q-TOF LC/MS G6520B system with a dual electrospray source (Agilent Technologies, Santa Clara, USA) were used to obtain mass spectra. The detector was set in a positive mode with the use of Agilent ESI-L (high-resolution mode-4 GHz). ${ }^{1} \mathrm{H}$ NMR spectra were recorded by Bruker Avance III $600 \mathrm{MHz}$ spectrometer, where tetramethylsilane was used as the internal standard.

\section{General procedure for the synthesis of compounds 2a-2h}

Step "a" in Scheme 1 was carried out to obtain compounds 2a-2h. 2-[1-(4-chlorobenzoyl)-5-methoxy-2-methylindol3 -yl]acetic acid (Indomethacin, Sigma-Aldrich) and N-methylmorpholine (as a water absorber) (Sigma-Aldrich) were added to a solution of 2-chloro-4,6-dimethoxy-1,3,5-triazine (CDMT, condensation agent, activator) (Sigma-Aldrich) in tetrahydrofuran $(10 \mathrm{~mL})$ (THF, as a solvent) (SigmaAldrich). The solution was stirred at room temperature for $3 \mathrm{~h}$. A solution of compounds 1a-1h dissolved in tetrahydrofuran (3 mL) (Sigma-Aldrich) (THF, as a solvent) was prepared. Both solutions were combined and stirred for $24 \mathrm{~h}$ at $-5^{\circ} \mathrm{C}$.

\section{$N$-(1,2,3,4-tetrahydro-9-acridinyl)-(2-aminoethyl)- 2-\{1-[(4-chlorophenyl)carbonyl]-5-methoxy-2-me- thyl-1H-indol-3-yl\}acetamide (2a)}

The solution of 2-[1-(4-chlorobenzoyl)-5-methoxy2-methylindol-3-yl]acetic acid $(0.12 \mathrm{~g} ; 0.34 \mathrm{mmol})$, 2-chloro-4,6-dimethoxy-1,3,5-triazine (CDMT) (0.06 g; $0.34 \mathrm{mmol}), N$ - methylmorpholine $(0.04 \mathrm{~mL} ; 0.36 \mathrm{mmol})$ and tetrahydrofuran (THF) $(10 \mathrm{~mL})$, the solution of
Scheme 1 Synthesis of $\mathbf{3 a}-\mathbf{3 h}$. Reagents: [a]-indomethacin, CDMT, $N$-methylmorpholine, $-5^{\circ} \mathrm{C}, 24 \mathrm{~h} ;[\mathrm{b}]-1.0 \mathrm{M} \mathrm{HCl}$ in diethyl ether, room temp., $24 \mathrm{~h}$

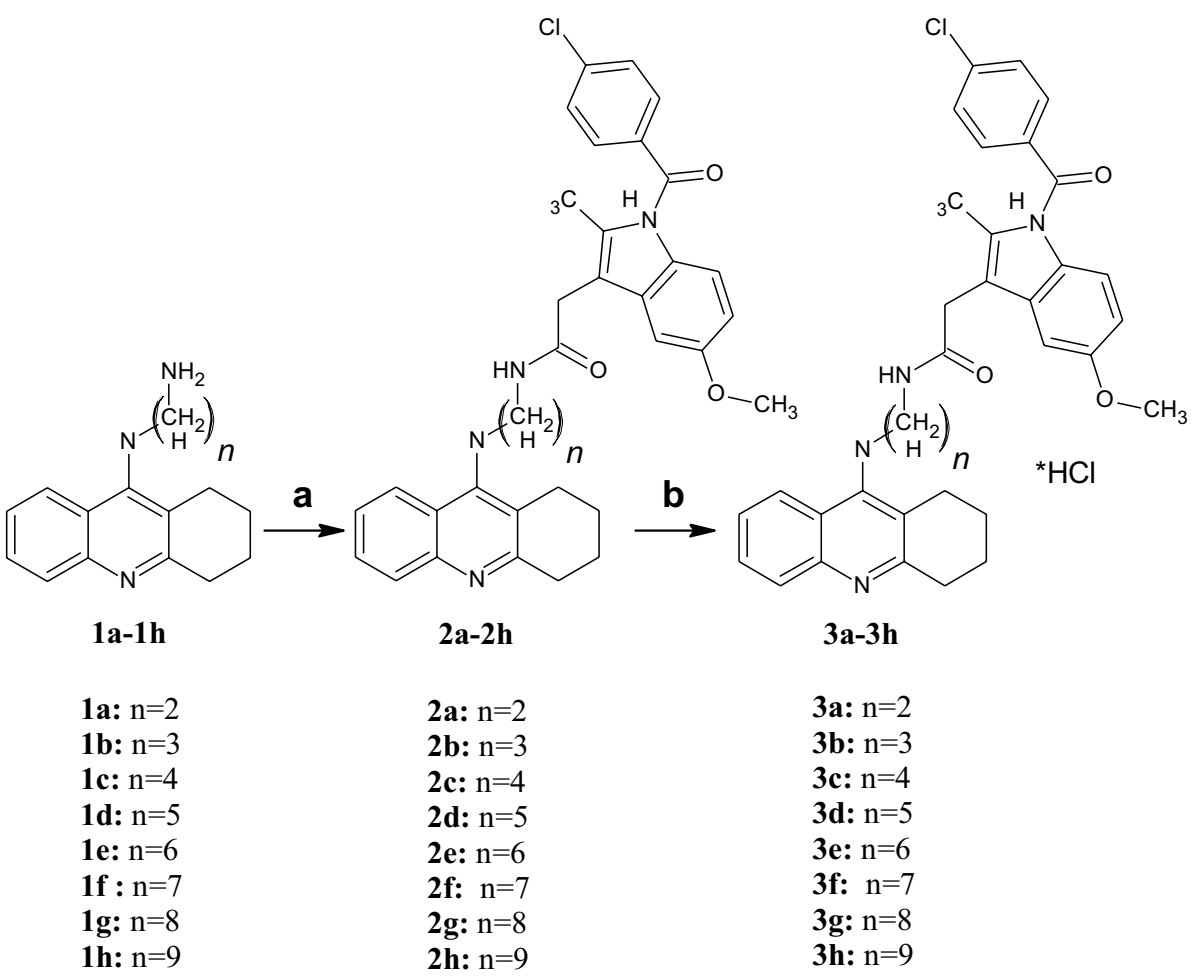


$N$-(1,2,3,4-tetrahydro-9-acrdinyl)-1,2-ethanediamine (0.08 g; $0.33 \mathrm{mmol}, \mathbf{1 a})$ dissolved in tetrahydrofuran (THF) ( $3 \mathrm{~mL}$ ) was added. Yield $12.58 \%$, orange solid; mp 163-165 ${ }^{\circ} \mathrm{C}$. FTIR (ATR) v $\left(\mathrm{cm}^{-1}\right)$ : 749.6, 1224.1, 1324.1, 1475.2, 1588.8, 2939.6, 3184.8; ${ }^{1} \mathrm{H}$ NMR ${ }^{1} \mathrm{H}$ NMR (600 MHz, Chloroform- $d) \delta 8.27(1 \mathrm{H}, \mathrm{d}, J=8.4 \mathrm{~Hz}$, Ar), $8.11(1 \mathrm{H}, \mathrm{d}, J=8.8 \mathrm{~Hz}, \mathrm{Ar}), 7.67-7.71$ (2H, m, Ar), 7.44-7.49 (3H, m, Ar), 7.25 (1H, t, J=7.9 Hz, Ar), 7.03 $(1 \mathrm{H}, \mathrm{s}, \mathrm{Ar}), 6.87(1 \mathrm{H}, \mathrm{d}, J=9.0 \mathrm{~Hz}, \mathrm{Ar}), 6.60-6.63(1 \mathrm{H}, \mathrm{m}$, Ar), 4.07-4.10 (2H, m, $\left.\mathrm{CH}_{2}\right), 4.06\left(3 \mathrm{H}, \mathrm{s}, \mathrm{CH}_{2}\right), 3.78(3 \mathrm{H}$, $\left.\mathrm{s}, \mathrm{CH}_{2}\right), 3.75\left(2 \mathrm{H}, \mathrm{q}, J=5.8 \mathrm{~Hz}, \mathrm{CH}_{2}\right), 3.52\left(1 \mathrm{H}, \mathrm{s}, \mathrm{CH}_{2}\right)$, $3.14\left(2 \mathrm{H}, \mathrm{t}, J=6.0 \mathrm{~Hz}, \mathrm{CH}_{2}\right), 2.48\left(1 \mathrm{H}, \mathrm{t}, J=5.9 \mathrm{~Hz}, \mathrm{CH}_{2}\right)$, 1.72-1.81 (4H, m, $\left.\mathrm{CH}_{2}\right)$; MS (ESI) $\mathrm{m} / z(\mathrm{M}+1)$ 581.2, 199.1, 143.0; MS-HR (ESI) Calcd for $\mathrm{C}_{34} \mathrm{H}_{33} \mathrm{ClN}_{4} \mathrm{O}_{3}: 580.22412$. Found: 580.22430 .

\section{$N$-(1,2,3,4-tetrahydro-9-acridinyl)-(3-aminopropyl) -2-\{1-[(4-chlorophenyl)carbonyl]-5-methoxy-2-me- thyl-1H-indol-3-yl\}acetamide (2b)}

The solution of 2-[1-(4-chlorobenzoyl)-5-methoxy-2-methylindol-3-yl]acetic acid $(0.38 \mathrm{~g} ; 1.06 \mathrm{mmol})$, 2-chloro4,6-dimethoxy-1,3,5-triazine (CDMT) (0.19 g; $1.08 \mathrm{mmol}$ ), $N$ - methylmorpholine $(0.12 \mathrm{~mL} ; 1.09 \mathrm{mmol})$ and tetrahydrofuran (THF) (10 mL), the solution of $N$-(1,2,3,4-tetrahydro9-acrdinyl)-1,3-propanediamine (0.27 g; $1.06 \mathrm{mmol}, \mathbf{1 b})$ dissolved in tetrahydrofuran (THF) $(3 \mathrm{~mL})$ was added. Yield 82.64\%, beige solid; $\mathrm{mp} 143-145^{\circ} \mathrm{C}$. FTIR (ATR) v $\left(\mathrm{cm}^{-1}\right)$ : 753.6, 1227.7, 1313.8, 1477.5, 1570.4, 2937.9, 3230.2; ${ }^{1} \mathrm{H}$ NMR (600 MHz, Chloroform-d) $\delta 8.49(1 \mathrm{H}, \mathrm{d}, J=8.3 \mathrm{~Hz}$, Ar), 8.18 (1H, d, J=8.6 Hz, Ar), 7.66-7.72 (3H, m, Ar), 7.49-7.52 (2H, m, Ar), 7.44 (1H, t, J=8.1 Hz, Ar), $7.00(1 \mathrm{H}$, s, Ar), $6.86(1 \mathrm{H}, \mathrm{d}, J=9.0 \mathrm{~Hz}, \mathrm{Ar}), 6.70(1 \mathrm{H}, \mathrm{d}, J=11.5 \mathrm{~Hz}$, Ar), 3.85-3.89 (2H, m, $\left.\mathrm{CH}_{2}\right), 3.82\left(3 \mathrm{H}, \mathrm{s}, \mathrm{CH}_{2}\right), 3.78(3 \mathrm{H}$, s, $\left.\mathrm{CH}_{2}\right), 3.52\left(1 \mathrm{H}, \mathrm{s}, \mathrm{CH}_{2}\right), 3.46\left(2 \mathrm{H}, \mathrm{q}, J=6.3 \mathrm{~Hz}, \mathrm{CH}_{2}\right)$, $3.31\left(2 \mathrm{H}, \mathrm{t}, J=6.3 \mathrm{~Hz}, \mathrm{CH}_{2}\right), 2.77\left(2 \mathrm{H}, \mathrm{t}, J=6.2 \mathrm{~Hz}, \mathrm{CH}_{2}\right)$, 1.86-1.98 (6H, m, $\left.\mathrm{CH}_{2}\right)$; MS (ESI) $m / z(\mathrm{M}+1)$ 595.2, 199.1, 143.0; MS-HR (ESI) Calcd for $\mathrm{C}_{35} \mathrm{H}_{35} \mathrm{ClN}_{4} \mathrm{O}_{3}: 594.23977$. Found: 594.24012.

\section{$N$-(1,2,3,4-tetrahydro-9-acridinyl)-(4-aminobutyl)- 2-\{1-[(4-chlorophenyl)carbonyl]-5-methoxy-2-me- thyl-1H-indol-3-yl\}acetamide (2c)}

The solution of 2-[1-(4-chlorobenzoyl)-5-methoxy-2-methylindol-3-yl]acetic acid $(0.16 \mathrm{~g} ; 0.45 \mathrm{mmol})$, 2-chloro4,6-dimethoxy-1,3,5-triazine (CDMT) (0.08 g; $0.45 \mathrm{mmol})$, $N$ - methylmorpholine $(0.05 \mathrm{~mL} ; 0.45 \mathrm{mmol})$ and tetrahydrofuran (THF) $(10 \mathrm{~mL})$, the solution of $N$-(1,2,3,4-tetrahydro-9-acrdinyl)-1,4-butanediamine $(0.12 \mathrm{~g}$; $0.45 \mathrm{mmol}$; 1c) dissolved in tetrahydrofuran (THF) $(3 \mathrm{~mL})$ was added. Yield $37.33 \%$, yellow solid; mp $132-134{ }^{\circ} \mathrm{C}$. FTIR (ATR) $\mathrm{v}\left(\mathrm{cm}^{-1}\right): 754.1,1224.2,1323.5,1474.6,1573.8,2938.7$,
3238.8; 1H NMR (600 MHz, Chloroform- $d$ ) $\delta 8.45(1 \mathrm{H}, \mathrm{d}$, $J=8.6 \mathrm{~Hz}, \mathrm{Ar}), 8.15(1 \mathrm{H}, \mathrm{d}, J=8.6 \mathrm{~Hz}, \mathrm{Ar}), 7.64-7.71(3 \mathrm{H}$, m, Ar), 7.49-7.52 (2H, m, Ar), 7.43 (1H, t, J=7.5 Hz, Ar), $6.98(1 \mathrm{H}, \mathrm{s}, \mathrm{Ar}), 6.86(1 \mathrm{H}, \mathrm{d}, J=9.0 \mathrm{~Hz}, \mathrm{Ar}), 6.67(1 \mathrm{H}, \mathrm{d}$, $J=11.5 \mathrm{~Hz}, \mathrm{Ar}), 3.87-3.92\left(2 \mathrm{H}, \mathrm{m}, \mathrm{CH}_{2}\right), 3.79\left(3 \mathrm{H}, \mathrm{s}, \mathrm{CH}_{2}\right)$, $3.71\left(3 \mathrm{H}, \mathrm{s}, \mathrm{CH}_{2}\right), 3.52\left(1 \mathrm{H}, \mathrm{s}, \mathrm{CH}_{2}\right), 3.34(2 \mathrm{H}, \mathrm{q}, J=6.6 \mathrm{~Hz}$, $\left.\mathrm{CH}_{2}\right), 3.27\left(2 \mathrm{H}, \mathrm{t}, J=6.2 \mathrm{~Hz}, \mathrm{CH}_{2}\right), 2.61(2 \mathrm{H}, \mathrm{t}, J=6.1 \mathrm{~Hz}$, $\left.\mathrm{CH}_{2}\right), 1.78-1.95\left(6 \mathrm{H}, \mathrm{m}, \mathrm{CH}_{2}\right), 1.62-1.69\left(2 \mathrm{H}, \mathrm{m}, \mathrm{CH}_{2}\right) ; \mathrm{MS}$ (ESI) $m / z(\mathrm{M}+1) 609.3,199.1,143.0$; MS-HR (ESI) Calcd for $\mathrm{C}_{36} \mathrm{H}_{37} \mathrm{ClN}_{4} \mathrm{O}_{3}$ : 608.25542. Found: 608.25646.

\section{$\mathrm{N}$-(1,2,3,4-tetrahydro-9-acridinyl)-(5-aminopentyl) -2-\{1-[(4-chlorophenyl)carbonyl]-5-methoxy-2-me- thyl-1H-indol-3-yl\}acetamide (2d)}

The solution of 2-[1-(4-chlorobenzoyl)-5-methoxy-2-methylindol-3-yl]acetic acid $(0.29 \mathrm{~g} ; 0.81 \mathrm{mmol}), 2$-chloro4,6-dimethoxy-1,3,5-triazine (CDMT) (0.14 g; $0.79 \mathrm{mmol})$, $N$ - methylmorpholine $(0.09 \mathrm{~mL} ; 0.82 \mathrm{mmol})$ and tetrahydrofuran (THF) (10 mL), the solution of $N$-(1,2,3,4-tetrahydro-9-acrdinyl)-1,5-pentanediamine (0.23 g; $0.81 \mathrm{mmol}$; 1d) dissolved in tetrahydrofuran (THF) $(3 \mathrm{~mL})$ was added. Yield $42.98 \%$, yellow solid; mp $134-136{ }^{\circ} \mathrm{C}$. FTIR (ATR) $\mathrm{v}\left(\mathrm{cm}^{-1}\right):$ 754.1, 1224.2, 1323.7, 1474.5, 1569.7, 2937.4, 3238.2; ${ }^{1} \mathrm{H}$ NMR (600 MHz, Chloroform-d) $\delta 8.54(1 \mathrm{H}, \mathrm{d}$, $J=7.8 \mathrm{~Hz}, \mathrm{Ar}), 8.18(1 \mathrm{H}, \mathrm{d}, J=8.6 \mathrm{~Hz}, \mathrm{Ar}), 7.66-7.73(3 \mathrm{H}$, $\mathrm{m}, \mathrm{Ar}), 7.49-7.52$ (2H, m, Ar), $7.45(1 \mathrm{H}, \mathrm{t}, J=8.1 \mathrm{~Hz}, \mathrm{Ar})$, $6.93(1 \mathrm{H}, \mathrm{s}, \mathrm{Ar}), 6.84(1 \mathrm{H}, \mathrm{d}, J=9.0 \mathrm{~Hz}, \mathrm{Ar}), 6.68(1 \mathrm{H}$, $\mathrm{d}, J=11.5 \mathrm{~Hz}, \mathrm{Ar}), 3.84-3.88\left(2 \mathrm{H}, \mathrm{m}, \mathrm{CH}_{2}\right), 3.80(3 \mathrm{H}, \mathrm{s}$, $\left.\mathrm{CH}_{2}\right), 3.69\left(3 \mathrm{H}, \mathrm{s}, \mathrm{CH}_{2}\right), 3.52\left(1 \mathrm{H}, \mathrm{s}, \mathrm{CH}_{2}\right), 3.33(2 \mathrm{H}, \mathrm{t}$, $\left.J=6.1 \mathrm{~Hz}, \mathrm{CH}_{2}\right), 3.29\left(2 \mathrm{H}, \mathrm{q}, J=6.6 \mathrm{~Hz}, \mathrm{CH}_{2}\right), 2.62(2 \mathrm{H}$, $\left.\mathrm{t}, J=6.2 \mathrm{~Hz}, \mathrm{CH}_{2}\right), 1.81-1.98\left(6 \mathrm{H}, \mathrm{m}, \mathrm{CH}_{2}\right), 1.55(2 \mathrm{H}, \mathrm{p}$, $\left.J=7.0 \mathrm{~Hz}, \mathrm{CH}_{2}\right), 1.43\left(2 \mathrm{H}, \mathrm{p}, J=7.7 \mathrm{~Hz}, \mathrm{CH}_{2}\right)$; MS (ESI) $m / z(\mathrm{M}+1) 623.3,199.1,143.0$; MS-HR (ESI) Calcd for $\mathrm{C}_{37} \mathrm{H}_{39} \mathrm{ClN}_{4} \mathrm{O}_{3}$ : 622.27107. Found: 622.27177.

\section{$N$-(1,2,3,4-tetrahydro-9-acridinyl)-(6-aminohexyl)- 2-\{1-[(4-chlorophenyl)carbonyl]-5-methoxy-2-me- thyl-1H-indol-3-yl\}acetamide (2e)}

The solution of 2-[1-(4-chlorobenzoyl)-5-methoxy-2-methylindol-3-yl]acetic acid $(0.30 \mathrm{~g} ; 0.84 \mathrm{mmol}), 2$-chloro4,6-dimethoxy-1,3,5-triazine (CDMT) (0.15 g; $0.85 \mathrm{mmol})$, $N$ - methylmorpholine $(0.09 \mathrm{~mL} ; 0.82 \mathrm{mmol})$ and tetrahydrofuran (THF) $(10 \mathrm{~mL})$, the solution of $N$-(1,2,3,4-tetrahydro-9-acrdinyl)-1,6-hexanediamine $(0.25 \mathrm{~g} ; 0.84 \mathrm{mmol}$; 1e) dissolved in tetrahydrofuran (THF) $(3 \mathrm{~mL})$ was added. Yield $15.59 \%$, brown solid; mp $145-147{ }^{\circ} \mathrm{C}$. FTIR (ATR) $\mathrm{v}\left(\mathrm{cm}^{-1}\right): 754.2,1224.1,1357.5,1474.6,1588.3,2932.1$, 3231.6; ${ }^{1} \mathrm{H}$ NMR (600 MHz, Chloroform-d) $\delta 8.54(1 \mathrm{H}$, $\mathrm{d}, J=8.7 \mathrm{~Hz}, \mathrm{Ar}), 8.17(1 \mathrm{H}, \mathrm{d}, J=8.6 \mathrm{~Hz}, \mathrm{Ar}), 7.67-7.75$ (3H, m, Ar), 7.44-7.53 (2H, m, Ar), 6.93 (1H, s, Ar), 6.86 $(1 \mathrm{H}, \mathrm{d}, J=9.0 \mathrm{~Hz}, \mathrm{Ar}), 6.70(1 \mathrm{H}, \mathrm{d}, J=11.5 \mathrm{~Hz}, \mathrm{Ar}), 3.86$ 
$\left(2 \mathrm{H}, \mathrm{q}, J=6.5,6.0 \mathrm{~Hz}, \mathrm{CH}_{2}\right), 3.83\left(3 \mathrm{H}, \mathrm{s}, \mathrm{CH}_{2}\right), 3.68(3 \mathrm{H}$, $\left.\mathrm{s}, \mathrm{CH}_{2}\right), 3.52\left(1 \mathrm{H}, \mathrm{s}, \mathrm{CH}_{2}\right), 3.34\left(2 \mathrm{H}, \mathrm{t}, J=6.6 \mathrm{~Hz}, \mathrm{CH}_{2}\right)$, $3.25\left(2 \mathrm{H}, \mathrm{q}, J=6.8 \mathrm{~Hz}, \mathrm{CH}_{2}\right), 2.61\left(2 \mathrm{H}, \mathrm{t}, J=6.3 \mathrm{~Hz}, \mathrm{CH}_{2}\right)$, 1.88-1.99 (4H, m, $\left.\mathrm{CH}_{2}\right), 1.77\left(2 \mathrm{H}, \mathrm{p}, J=7.3 \mathrm{~Hz}, \mathrm{CH}_{2}\right)$, 1.43-1.52 (4H, m, 6H), 1.30-1.35 (2H, m, $\left.\mathrm{CH}_{2}\right)$; MS (ESI) $\mathrm{m} / \mathrm{z}(\mathrm{M}+1)$ 637.3, 308.2, 198.1, 143.0; MS-HR (ESI) Calcd for $\mathrm{C}_{38} \mathrm{H}_{41} \mathrm{ClN}_{4} \mathrm{O}_{3}$ : 636.28672. Found: 636.28742.

\section{$N$-(1,2,3,4-tetrahydro-9-acridinyl)-(7-aminoheptyl) -2-\{1-[(4-chlorophenyl)carbonyl]-5-methoxy-2-me- thyl-1H-indol-3-yl\}acetamide (2f)}

The solution of 2-[1-(4-chlorobenzoyl)-5-methoxy-2-methylindol-3-yl]acetic acid $(0.51 \mathrm{~g} ; 1.43 \mathrm{mmol})$, 2-chloro4,6-dimethoxy-1,3,5-triazine (CDMT) (0.25 g; $1.42 \mathrm{mmol})$, $N$ - methylmorpholine $(0.15 \mathrm{~mL} ; 1.36 \mathrm{mmol})$ and tetrahydrofuran (THF) $(10 \mathrm{~mL})$, the solution of $N$-(1,2,3,4-tetrahydro-9-acrdinyl)-1,7-heptanediamine (0.44 g; $1.41 \mathrm{mmol}$; 1f) dissolved in tetrahydrofuran (THF) ( $3 \mathrm{~mL})$ was added. Yield 42.48\%, yellow solid; mp $119-121{ }^{\circ} \mathrm{C}$. FTIR (ATR) $\mathrm{v}\left(\mathrm{cm}^{-1}\right): 753.9,1224.5,1325.4,1473.8,1576.8,2932.1$, 3231.3; ${ }^{1} \mathrm{H}$ NMR (600 MHz, Chloroform-d) $\delta 8.56(1 \mathrm{H}$, d, $J=8.3 \mathrm{~Hz}, \mathrm{Ar}), 8.16(1 \mathrm{H}, \mathrm{d}, J=8.6 \mathrm{~Hz}, \mathrm{Ar}), 7.68-7.74$ (3H, m, Ar), 7.50-7.53 (2H, m, Ar), 7.47 (1H, t, J=7.8 Hz, Ar), 6.92 (1H, s, Ar), 6.85-6.88 (1H, m, Ar), $6.71(1 \mathrm{H}, \mathrm{d}$, $J=11.5 \mathrm{~Hz}, \mathrm{Ar}), 3.86-3.90\left(2 \mathrm{H}, \mathrm{m}, \mathrm{CH}_{2}\right), 3.83\left(3 \mathrm{H}, \mathrm{s}, \mathrm{CH}_{2}\right)$, $3.67\left(3 \mathrm{H}, \mathrm{s}, \mathrm{CH}_{2}\right), 3.52\left(1 \mathrm{H}, \mathrm{s}, \mathrm{CH}_{2}\right), 3.33-3.38(2 \mathrm{H}, \mathrm{m}$, $\left.\mathrm{CH}_{2}\right), 3.23\left(2 \mathrm{H}, \mathrm{q}, J=6.8 \mathrm{~Hz}, \mathrm{CH}_{2}\right), 2.61(2 \mathrm{H}, \mathrm{t}, J=6.2 \mathrm{~Hz}$, $\left.\mathrm{CH}_{2}\right), 1.88-1.99\left(4 \mathrm{H}, \mathrm{m}, \mathrm{CH}_{2}\right), 1.82-1.75\left(2 \mathrm{H}, \mathrm{m}, \mathrm{CH}_{2}\right)$, 1.47-1.30 (8H, m, $\left.\mathrm{CH}_{2}\right)$; MS (ESI) $\mathrm{m} / \mathrm{z}(\mathrm{M}+1)$ 651.3, 322.2, 174.1, 143.0; MS-HR (ESI) Calcd for $\mathrm{C}_{39} \mathrm{H}_{43} \mathrm{ClN}_{4} \mathrm{O}_{3}$ : 650.30237. Found: 650.30350 .

\section{$N$-(1,2,3,4-tetrahydro-9-acridinyl)-(8-aminooctyl)- 2-\{1-[(4-chlorophenyl)carbonyl]-5-methoxy-2-me- thyl-1H-indol-3-yl\}acetamide (2g)}

The solution of 2-[1-(4-chlorobenzoyl)-5-methoxy-2-methylindol-3-yl]acetic acid (0.48 g; $1.34 \mathrm{mmol})$, 2-chloro4,6-dimethoxy-1,3,5-triazine (CDMT) (0.24 g; $1.36 \mathrm{mmol})$, $N$ - methylmorpholine $(0.15 \mathrm{~mL} ; 1.36 \mathrm{mmol})$ and tetrahydrofuran (THF) (10 mL), the solution of $N$-(1,2,3,4-tetrahydro9-acrdinyl)-1,8-octanediamine ( $0.44 \mathrm{~g} ; 1.35 \mathrm{mmol}$; 1g) dissolved in tetrahydrofuran (THF) $(3 \mathrm{~mL})$ was added. Yield $48.29 \%$, beige solid; $\mathrm{mp} 122-124^{\circ} \mathrm{C}$. FTIR (ATR) v $\left(\mathrm{cm}^{-1}\right)$ : 754.1, 1224.7, 1325.1, 1473.0, 1568.8, 2927.5, 3227.8; ${ }^{1} \mathrm{H}$ NMR (600 MHz, Chloroform- $d$ ) $\delta 8.55(1 \mathrm{H}, \mathrm{d}, J=8.5 \mathrm{~Hz}$, Ar), $8.16(1 \mathrm{H}, \mathrm{d}, J=8.6 \mathrm{~Hz}, \mathrm{Ar}), 7.67-7.75$ (3H, m, Ar), 7.49-7.53 (2H, m, Ar), 7.47 (1H, t, J=7.7 Hz, Ar), $6.91(1 \mathrm{H}$, $\mathrm{s}, \mathrm{Ar}), 6.87(1 \mathrm{H}, \mathrm{d}, J=9.0 \mathrm{~Hz}, \mathrm{Ar}), 6.71(1 \mathrm{H}, \mathrm{d}, J=11.5 \mathrm{~Hz}$, Ar), $3.87\left(2 \mathrm{H}, \mathrm{t}, J=7.2 \mathrm{~Hz}, \mathrm{CH}_{2}\right), 3.84\left(3 \mathrm{H}, \mathrm{s}, \mathrm{CH}_{2}\right), 3.67$ $\left(3 \mathrm{H}, \mathrm{s}, \mathrm{CH}_{2}\right), 3.52\left(1 \mathrm{H}, \mathrm{s}, \mathrm{CH}_{2}\right), 3.33-3.37\left(2 \mathrm{H}, \mathrm{m}, \mathrm{CH}_{2}\right)$, $3.22\left(2 \mathrm{H}, \mathrm{q}, J=6.9 \mathrm{~Hz}, \mathrm{CH}_{2}\right), 2.61\left(2 \mathrm{H}, \mathrm{t}, J=6.3 \mathrm{~Hz}, \mathrm{CH}_{2}\right)$,
1.89-2.00 (4H, m, $\left.\mathrm{CH}_{2}\right), 1.77-1.82\left(2 \mathrm{H}, \mathrm{m}, \mathrm{CH}_{2}\right), 1.39-1.46$ $\left(4 \mathrm{H}, \mathrm{m}, \mathrm{CH}_{2}\right), 1.29-1.35\left(4 \mathrm{H}, \mathrm{m}, \mathrm{CH}_{2}\right) ; \mathrm{MS}(\mathrm{ESI}) \mathrm{m} / \mathrm{z}$ (M+1) 665.3, 336.2, 174.1, 143.0; MS-HR (ESI) Calcd for $\mathrm{C}_{40} \mathrm{H}_{45} \mathrm{ClN}_{4} \mathrm{O}_{3}$ : 664.31802. Found: 664.31861.

\section{$N$-(1,2,3,4-tetrahydro-9-acridinyl)-(9-aminononyl)- 2-\{1-[(4-chlorophenyl)carbonyl]-5-methoxy-2-me- thyl-1H-indol-3-yl\}acetamide (2h)}

The solution of 2-[1-(4-chlorobenzoyl)-5-methoxy-2-methylindol-3-yl]acetic acid (0.68 g; $1.9 \mathrm{mmol})$, 2-chloro4,6-dimethoxy-1,3,5-triazine (CDMT) (0.34 g; $1.93 \mathrm{mmol})$, $N$ - methylmorpholine $(0.21 \mathrm{~mL} ; 1.91 \mathrm{mmol})$ and tetrahydrofuran (THF) $(10 \mathrm{~mL})$, the solution of N-(1,2,3,4-tetrahydro9-acrdinyl)-1,9-nonanediamine (0.65 g; $1.91 \mathrm{mmol}$; $1 \mathbf{h})$ dissolved in tetrahydrofuran (THF) $(3 \mathrm{~mL})$ was added. Yield $17.38 \%$, brown solid; $\mathrm{mp} 96-98^{\circ} \mathrm{C}$. FTIR (ATR) v $\left(\mathrm{cm}^{-1}\right)$ : 754.3, 1219.5, 1355.1, 1471.4, 1587.44, 2926.6, 3238.1; ${ }^{1} \mathrm{H}{ }^{1} \mathrm{H}$ NMR (600 MHz, Chloroform-d) $\delta 8.56(1 \mathrm{H}, \mathrm{d}$, $J=9.3 \mathrm{~Hz}, \mathrm{Ar}), 8.16(1 \mathrm{H}, \mathrm{d}, J=8.6 \mathrm{~Hz}, \mathrm{Ar}), 7.71-7.76(3 \mathrm{H}$, $\mathrm{m}, \mathrm{Ar}), 7.69$ (1H, d, $J=8.5 \mathrm{~Hz}, \mathrm{Ar}), 7.45-7.53$ (2H, m, Ar), $6.92(1 \mathrm{H}, \mathrm{s}, \mathrm{Ar}), 6.88(1 \mathrm{H}, \mathrm{d}, J=9.0 \mathrm{~Hz}, \mathrm{Ar}), 6.71(1 \mathrm{H}, \mathrm{d}$, $J=11.5 \mathrm{~Hz}, \mathrm{Ar}), 3.86-3.91\left(2 \mathrm{H}, \mathrm{m}, \mathrm{CH}_{2}\right), 3.84\left(3 \mathrm{H}, \mathrm{s}, \mathrm{CH}_{2}\right)$, $3.67\left(3 \mathrm{H}, \mathrm{s}, \mathrm{CH}_{2}\right), 3.52\left(1 \mathrm{H}, \mathrm{s}, \mathrm{CH}_{2}\right), 3.35(2 \mathrm{H}, \mathrm{t}, J=5.8 \mathrm{~Hz}$, $\left.\mathrm{CH}_{2}\right), 3.21\left(2 \mathrm{H}, \mathrm{q}, J=6.8 \mathrm{~Hz}, \mathrm{CH}_{2}\right), 2.61(2 \mathrm{H}, \mathrm{t}, J=6.2 \mathrm{~Hz}$, $\mathrm{CH}_{2}$ ), 1.89-2.00 (4H, m, $\left.\mathrm{CH}_{2}\right), 1.76-1.84\left(2 \mathrm{H}, \mathrm{m}, \mathrm{CH}_{2}\right)$, 1.56-1.64 (4H, m, $\left.\mathrm{CH}_{2}\right), 1.32-1.54\left(6 \mathrm{H}, \mathrm{m}, \mathrm{CH}_{2}\right)$; MS (ESI) $m / z(\mathrm{M}+1) 679.3,350.3,184.0,174.1 ; \mathrm{MS}-\mathrm{HR}$ (ESI) Calcd for $\mathrm{C}_{41} \mathrm{H}_{47} \mathrm{ClN}_{4} \mathrm{O}_{3}$ : 678.33367. Found: 678.33401.

\section{General procedure for synthesis compounds $3 a-3 h$}

For improve solubility, compounds $\mathbf{2 a - 2 h}$ were converted into hydrochlorides. Step "b" in Scheme 1 was carried out to obtain compounds $\mathbf{3 a - 3 h}$. The solutions of compound $\mathbf{2 a - 2 h}$ dissolved in methanol (1 mL) (POCH) was treated with excess $1.0 \mathrm{M} \mathrm{HCl}$ in diethyl ether (4 mL) (Sigma Aldrich). The mixture was stirred for $24 \mathrm{~h}$ at room temperature to crystallize the hydrochloride form which was isolated by decantation and dried.

\section{$N$-(1,2,3,4-tetrahydro-9-acridinyl)-(2-aminoethyl)- 2-\{1-[(4-chlorophenyl)carbonyl]-5-methoxy-2-me- thyl-1H-indol-3-yl\}acetamide hydrochloride (3a)}

To solution of $2 \mathbf{a}(0.20 \mathrm{~g} ; 0.34 \mathrm{mmol})$ in methanol $(1 \mathrm{~mL})$ $1.0 \mathrm{M} \mathrm{HCl}$ in diethyl ether $(4 \mathrm{~mL})$ was added in excess. Yield $80.77 \%$, orange solid, mp $182-184{ }^{\circ} \mathrm{C}$. FTIR (ATR) $\mathrm{V}\left(\mathrm{cm}^{-1}\right):$ 757.0, 1231.4, 1323.7, 1475.2, 1578.5, 2944.1, 3215.8; ${ }^{1} \mathrm{H}$ NMR $\left(600 \mathrm{MHz}\right.$, Methanol- $\left.d_{4}\right) \delta 8.34(1 \mathrm{H}, \mathrm{d}$, $J=8.9 \mathrm{~Hz}, \mathrm{Ar}), 7.84(1 \mathrm{H}, \mathrm{t}, J=8.2 \mathrm{~Hz}, \mathrm{Ar}), 7.68-7.73(3 \mathrm{H}$, $\mathrm{m}, \mathrm{Ar}), 7.57-7.61(2 \mathrm{H}, \mathrm{m}, \mathrm{Ar}), 7.52(1 \mathrm{H}, \mathrm{t}, J=8.4 \mathrm{~Hz}, \mathrm{Ar})$, $6.96(1 \mathrm{H}, \mathrm{d}, J=9.0 \mathrm{~Hz}, \mathrm{Ar}), 6.76(1 \mathrm{H}, \mathrm{s}, \mathrm{Ar}), 6.56(1 \mathrm{H}$, 
d, $J=11.5 \mathrm{~Hz}, \mathrm{Ar}), 4.16-4.20\left(2 \mathrm{H}, \mathrm{m}, \mathrm{CH}_{2}\right), 3.78(2 \mathrm{H}, \mathrm{q}$, $\left.J=5.9 \mathrm{~Hz}, \mathrm{CH}_{2}\right), 3.62\left(3 \mathrm{H}, \mathrm{s}, \mathrm{CH}_{2}\right), 3.41\left(2 \mathrm{H}, \mathrm{s}, \mathrm{CH}_{2}\right), 2.85$ $\left(2 \mathrm{H}, \mathrm{t}, J=6.2 \mathrm{~Hz}, \mathrm{CH}_{2}\right), 2.31-2.36\left(2 \mathrm{H}, \mathrm{m}, \mathrm{CH}_{2}\right), 1.66-1.74$ (4H, m, $\mathrm{CH}_{2}$ ); MS (ESI) $\mathrm{m} / \mathrm{z}(\mathrm{M}+1) 581.2,201.0,143.0$, 81.0; MS-HR (ESI) Calcd for $\mathrm{C}_{34} \mathrm{H}_{33} \mathrm{ClN}_{4} \mathrm{O}_{3}: 580.22412$. Found: 580.22391.

\section{$\mathrm{N}$-(1,2,3,4-tetrahydro-9-acridinyl)-(3-aminopropyl) -2-\{1-[(4-chlorophenyl)carbonyl]-5-methoxy-2-me- thyl-1H-indol-3-yl\}acetamide hydrochloride (3b)}

To solution of $\mathbf{2 b}(0.20 \mathrm{~g}$; $0.34 \mathrm{mmol})$ in methanol $(1 \mathrm{~mL})$ $1.0 \mathrm{M} \mathrm{HCl}$ in diethyl ether $(4 \mathrm{~mL})$ was added in excess. Yield $86.54 \%$, beige solid, mp $160-162{ }^{\circ} \mathrm{C}$. FTIR (ATR) $\mathrm{v}\left(\mathrm{cm}^{-1}\right)$ : 756.1, 1230.2, 1322.7, 1474.5, 1578.1, 2942.2, 3216.3 ; ${ }^{1} \mathrm{H}$ NMR $\left(600 \mathrm{MHz}\right.$, Methanol- $\left.d_{4}\right) \delta 8.27(1 \mathrm{H}, \mathrm{d}$, $J=8.5 \mathrm{~Hz}, \mathrm{Ar}), 7.83(1 \mathrm{H}, \mathrm{d}, J=7.3 \mathrm{~Hz}, \mathrm{Ar}), 7.69-7.74(3 \mathrm{H}$, $\mathrm{m}, \mathrm{Ar}), 7.57-7.60(2 \mathrm{H}, \mathrm{m}, \mathrm{Ar}), 7.49(1 \mathrm{H}, \mathrm{t}, J=8.2 \mathrm{~Hz}, \mathrm{Ar})$, $7.00(1 \mathrm{H}, \mathrm{s}, \mathrm{Ar}), 6.86(1 \mathrm{H}, \mathrm{d}, J=9.0 \mathrm{~Hz}, \mathrm{Ar}), 6.60(1 \mathrm{H}$, $\mathrm{d}, J=9.0 \mathrm{~Hz}, \mathrm{Ar}), 3.87-3.90\left(2 \mathrm{H}, \mathrm{m}, \mathrm{CH}_{2}\right), 3.73(3 \mathrm{H}, \mathrm{s}$, $\left.\mathrm{CH}_{2}\right), 3.62\left(1 \mathrm{H}, \mathrm{s}, \mathrm{CH}_{2}\right), 3.43\left(2 \mathrm{H}, \mathrm{q}, J=5.9 \mathrm{~Hz}, \mathrm{CH}_{2}\right), 3.01$ $\left(1 \mathrm{H}, \mathrm{t}, J=5.2 \mathrm{~Hz}, \mathrm{CH}_{2}\right), 2.66-2.69\left(2 \mathrm{H}, \mathrm{m}, \mathrm{CH}_{2}\right), 2.03(2 \mathrm{H}$, p, $\left.J=6.6 \mathrm{~Hz}, \mathrm{CH}_{2}\right), 1.91-1.97\left(4 \mathrm{H}, \mathrm{m}, \mathrm{CH}_{2}\right)$; MS (ESI) $\mathrm{m} / \mathrm{z}(\mathrm{M}+1) 595.2,199.1,143.0$; MS-HR (ESI) Calcd for $\mathrm{C}_{35} \mathrm{H}_{35} \mathrm{ClN}_{4} \mathrm{O}_{3}$ : 594.23977. Found: 594.24029.

\section{$N$-(1,2,3,4-tetrahydro-9-acridinyl)-(4-aminobutyl)- 2-\{1-[(4-chlorophenyl)carbonyl]-5-methoxy-2-me- thyl-1H-indol-3-yl\}acetamide hydrochloride (3c)}

To solution of $2 \mathbf{c}(0.20 \mathrm{~g} ; 0.33 \mathrm{mmol})$ in methanol $(1 \mathrm{~mL})$ $1.0 \mathrm{M} \mathrm{HCl}$ in diethyl ether $(4 \mathrm{~mL})$ was added in excess. Yield $92.31 \%$, yellow solid, mp $157-159^{\circ} \mathrm{C}$. FTIR (ATR) $\mathrm{v}\left(\mathrm{cm}^{-1}\right)$ : 756.3, 1219.7, 1323.4, 1473.8, 1576.9, 2941.4, 3223.2; ${ }^{1} \mathrm{H}$ NMR $\left(600 \mathrm{MHz}\right.$, Methanol- $\left.d_{4}\right) \delta 8.24(1 \mathrm{H}, \mathrm{d}$, $J=8.7 \mathrm{~Hz}, \mathrm{Ar}), 7.83(1 \mathrm{H}, \mathrm{t}, J=7.7 \mathrm{~Hz}, \mathrm{Ar}), 7.74(1 \mathrm{H}, \mathrm{d}$, $J=9.3 \mathrm{~Hz}, \mathrm{Ar}), 7.67-7.70(2 \mathrm{H}, \mathrm{m}, \mathrm{Ar}), 7.56-7.60(2 \mathrm{H}, \mathrm{m}$, Ar), $7.52(1 \mathrm{H}, \mathrm{t}, J=8.4 \mathrm{~Hz}, \mathrm{Ar}), 7.00(1 \mathrm{H}, \mathrm{s}, \mathrm{Ar}), 6.84(1 \mathrm{H}$, d, $J=9.0 \mathrm{~Hz}, \mathrm{Ar}), 6.53-6.56(1 \mathrm{H}, \mathrm{m}, \mathrm{Ar}), 3.88-3.92(2 \mathrm{H}$, $\left.\mathrm{m}, \mathrm{CH}_{2}\right), 3.71\left(3 \mathrm{H}, \mathrm{s}, \mathrm{CH}_{2}\right), 3.60\left(1 \mathrm{H}, \mathrm{s}, \mathrm{CH}_{2}\right), 3.31(2 \mathrm{H}, \mathrm{t}$, $\left.J=6.7 \mathrm{~Hz}, \mathrm{CH}_{2}\right), 3.01\left(2 \mathrm{H}, \mathrm{t}, J=5.4 \mathrm{~Hz}, \mathrm{CH}_{2}\right), 2.62(2 \mathrm{H}$, t, $\left.J=5.3 \mathrm{~Hz}, \mathrm{CH}_{2}\right), 1.91-1.97\left(4 \mathrm{H}, \mathrm{m}, \mathrm{CH}_{2}\right), 1.80(2 \mathrm{H}, \mathrm{p}$, $\left.J=8.1,7.7 \mathrm{~Hz}, \mathrm{CH}_{2}\right), 1.63-1.69\left(2 \mathrm{H}, \mathrm{m}, \mathrm{CH}_{2}\right) ; \mathrm{MS}(\mathrm{ESI})$ $\mathrm{m} / \mathrm{z}(\mathrm{M}+1) 609.3,199.1,143.0$; MS-HR (ESI) Calcd for $\mathrm{C}_{36} \mathrm{H}_{37} \mathrm{ClN}_{4} \mathrm{O}_{3}: 608.25542$. Found: 608.25597.

\section{$N$-(1,2,3,4-tetrahydro-9-acridinyl)-(5-aminopentyl) -2-\{1-[(4-chlorophenyl)carbonyl]-5-methoxy-2-me- thyl-1H-indol-3-yl\}acetamide hydrochloride (3d)}

To solution of $\mathbf{2 d}(0.20 \mathrm{~g}$; $0.32 \mathrm{mmol})$ in methanol $(1 \mathrm{~mL})$ $1.0 \mathrm{M} \mathrm{HCl}$ in diethyl ether $(4 \mathrm{~mL})$ was added in excess. Yield $87.50 \%$, yellow solid, mp $158-160^{\circ} \mathrm{C}$. FTIR (ATR) v $\left(\mathrm{cm}^{-1}\right): 754.5,1223.8,1323.2,1455.4,1587.5,2937.7$, 3207.7; ${ }^{1} \mathrm{H}$ NMR $\left(600 \mathrm{MHz}\right.$, Methanol- $\left.d_{4}\right) \delta 8.33(1 \mathrm{H}, \mathrm{d}$, $J=8.5 \mathrm{~Hz}, \mathrm{Ar}), 7.85(1 \mathrm{H}, \mathrm{t}, J=8.2 \mathrm{~Hz}, \mathrm{Ar}), 7.75(1 \mathrm{H}, \mathrm{d}$, $J=9.3 \mathrm{~Hz}, \mathrm{Ar}), 7.67-7.70(2 \mathrm{H}, \mathrm{m}, \mathrm{Ar}), 7.54-7.59(3 \mathrm{H}, \mathrm{m}$, Ar), $7.03(1 \mathrm{H}, \mathrm{s}, \mathrm{Ar}), 6.89(1 \mathrm{H}, \mathrm{d}, J=9.0 \mathrm{~Hz}, \mathrm{Ar}), 6.60(1 \mathrm{H}$, d, $J=11.5 \mathrm{~Hz}, \mathrm{Ar}), 3.82-3.86\left(2 \mathrm{H}, \mathrm{m}, \mathrm{CH}_{2}\right), 3.77(3 \mathrm{H}, \mathrm{s}$, $\left.\mathrm{CH}_{2}\right), 3.61\left(1 \mathrm{H}, \mathrm{s}, \mathrm{CH}_{2}\right), 3.25-3.28\left(2 \mathrm{H}, \mathrm{m}, \mathrm{CH}_{2}\right), 3.02(2 \mathrm{H}$, $\left.\mathrm{t}, J=5.7 \mathrm{~Hz}, \mathrm{CH}_{2}\right), 2.65-2.69\left(2 \mathrm{H}, \mathrm{m}, \mathrm{CH}_{2}\right), 2.00-1.93$ $\left(4 \mathrm{H}, \mathrm{m}, \mathrm{CH}_{2}\right), 1.83\left(2 \mathrm{H}, \mathrm{p}, J=7.6 \mathrm{~Hz}, \mathrm{CH}_{2}\right), 1.60(2 \mathrm{H}, \mathrm{p}$, $\left.J=7.0 \mathrm{~Hz}, \mathrm{CH}_{2}\right), 1.42\left(2 \mathrm{H}, \mathrm{p}, J=9.3,8.6 \mathrm{~Hz}, \mathrm{CH}_{2}\right) ; \mathrm{MS}$ (ESI) $\mathrm{m} / z(\mathrm{M}+1) 623.3,199.1,143.0$; MS-HR (ESI) Calcd for $\mathrm{C}_{37} \mathrm{H}_{39} \mathrm{ClN}_{4} \mathrm{O}_{3}$ : 622.27107. Found: 622.27072 .

\section{$N$-(1,2,3,4-tetrahydro-9-acridinyl)-(6-aminohexyl)- 2-\{1-[(4-chlorophenyl)carbonyl]-5-methoxy-2-me- thyl-1H-indol-3-yl\}acetamide hydrochloride (3e)}

To solution of $2 \mathbf{e}(0.20 \mathrm{~g} ; 0.31 \mathrm{mmol})$ in methanol $(1 \mathrm{~mL})$ $1.0 \mathrm{M} \mathrm{HCl}$ in diethyl ether $(4 \mathrm{~mL})$ was added in excess. Yield $56.00 \%$, brown solid, $\mathrm{mp} 83-85^{\circ} \mathrm{C}$. FTIR (ATR) v $\left(\mathrm{cm}^{-1}\right)$ : 754.4, 1218.9, 1324.2, 1456.0, 1587.1, 2934.9, 3237.8; ${ }^{1} \mathrm{H}$ NMR $\left(600 \mathrm{MHz}\right.$, Methanol- $\left.d_{4}\right) \delta 8.30(1 \mathrm{H}, \mathrm{d}, J=8.5 \mathrm{~Hz}$, $\mathrm{Ar}), 7.82-7.87(1 \mathrm{H}, \mathrm{m}, \mathrm{Ar}), 7.75(1 \mathrm{H}, \mathrm{d}, J=7.7 \mathrm{~Hz}, \mathrm{Ar})$, 7.64-7.67 (2H, m, Ar), 7.54-7.58 (3H, m, Ar), $7.05(1 \mathrm{H}, \mathrm{s}$, $\mathrm{Ar}), 6.86(1 \mathrm{H}, \mathrm{d}, J=9.0 \mathrm{~Hz}, \mathrm{Ar}), 6.59(1 \mathrm{H}, \mathrm{d}, J=11.5 \mathrm{~Hz}$, Ar), 3.79-3.83 $\left(2 \mathrm{H}, \mathrm{m}, \mathrm{CH}_{2}\right), 3.78\left(3 \mathrm{H}, \mathrm{s}, \mathrm{CH}_{2}\right), 3.61(2 \mathrm{H}$, $\left.\mathrm{s}, \mathrm{CH}_{2}\right), 3.24-3.28\left(2 \mathrm{H}, \mathrm{m}, \mathrm{CH}_{2}\right), 3.02(2 \mathrm{H}, \mathrm{t}, J=5.4 \mathrm{~Hz}$, $\left.\mathrm{CH}_{2}\right), 2.66\left(2 \mathrm{H}, \mathrm{t}, J=5.1 \mathrm{~Hz}, \mathrm{CH}_{2}\right), 1.95-2.00\left(4 \mathrm{H}, \mathrm{m}, \mathrm{CH}_{2}\right)$, $1.74\left(2 \mathrm{H}, \mathrm{p}, J=7.4 \mathrm{~Hz}, \mathrm{CH}_{2}\right), 1.55\left(2 \mathrm{H}, \mathrm{p}, J=7.0 \mathrm{~Hz}, \mathrm{CH}_{2}\right)$, 1.40-1.46 (2H, m, $\left.\mathrm{CH}_{2}\right), 1.38-1.31\left(2 \mathrm{H}, \mathrm{m}, \mathrm{CH}_{2}\right)$; $\mathrm{MS}(\mathrm{ESI})$ $\mathrm{m} / \mathrm{z}(\mathrm{M}+1) 637.3,308.2,174.1,143.0$; MS-HR (ESI) Calcd for $\mathrm{C}_{38} \mathrm{H}_{41} \mathrm{ClN}_{4} \mathrm{O}_{3}$ : 636.28672. Found: 636.28658.

\section{$\mathrm{N}$-(1,2,3,4-tetrahydro-9-acridinyl)-(7-aminoheptyl) -2-\{1-[(4-chlorophenyl)carbonyl]-5-methoxy-2-me- thyl-1H-indol-3-yl\}acetamide hydrochloride (3f)}

To solution of $\mathbf{2 f}(0.20 \mathrm{~g}$; $0.31 \mathrm{mmol})$ in methanol $(1 \mathrm{~mL})$ $1.0 \mathrm{M} \mathrm{HCl}$ in diethyl ether $(4 \mathrm{~mL})$ was added in excess. Yield $72.00 \%$, yellow solid, mp $150-152{ }^{\circ} \mathrm{C}$. FTIR (ATR) $\mathrm{v}\left(\mathrm{cm}^{-1}\right): 754.5,1224.4,1323.5,1456.1,1587.5,2931.3$, $3207.8 ;{ }^{1} \mathrm{H}$ NMR $\left(600 \mathrm{MHz}\right.$, Methanol- $\left.d_{4}\right) \delta 8.36(1 \mathrm{H}, \mathrm{d}$, $J=8.6 \mathrm{~Hz}, \mathrm{Ar}), 7.86(1 \mathrm{H}, \mathrm{t}, J=7.7 \mathrm{~Hz}, \mathrm{Ar}), 7.76(1 \mathrm{H}, \mathrm{d}$, $J=9.3 \mathrm{~Hz}, \mathrm{Ar}), 7.67-7.70(2 \mathrm{H}, \mathrm{m}, \mathrm{Ar}), 7.55-7.60(3 \mathrm{H}, \mathrm{m}$, Ar), $7.04(1 \mathrm{H}, \mathrm{s}, \mathrm{Ar}), 6.89-6.92(1 \mathrm{H}, \mathrm{m}, \mathrm{Ar}), 6.63(1 \mathrm{H}, \mathrm{d}$, $J=11.5 \mathrm{~Hz}, \mathrm{Ar}), 3.87-3.91\left(2 \mathrm{H}, \mathrm{m}, \mathrm{CH}_{2}\right), 3.80\left(3 \mathrm{H}, \mathrm{s}, \mathrm{CH}_{2}\right)$, $3.61\left(2 \mathrm{H}, \mathrm{s}, \mathrm{CH}_{2}\right), 3.21-3.25\left(2 \mathrm{H}, \mathrm{m}, \mathrm{CH}_{2}\right), 3.03(2 \mathrm{H}, \mathrm{t}$, $\left.J=5.6 \mathrm{~Hz}, \mathrm{CH}_{2}\right), 2.69\left(2 \mathrm{H}, \mathrm{t}, J=5.3 \mathrm{~Hz}, \mathrm{CH}_{2}\right), 1.95-2.00$ $\left(4 \mathrm{H}, \mathrm{m}, \mathrm{CH}_{2}\right), 1.77\left(2 \mathrm{H}, \mathrm{p}, J=7.5 \mathrm{~Hz}, \mathrm{CH}_{2}\right), 1.52(2 \mathrm{H}, \mathrm{p}$, $\left.J=6.9 \mathrm{~Hz}, \mathrm{CH}_{2}\right), 1.27-1.39\left(6 \mathrm{H}, \mathrm{m}, \mathrm{CH}_{2}\right) ; \mathrm{MS}(\mathrm{ESI}) \mathrm{m} / \mathrm{z}$ $(\mathrm{M}+1)$ 651.3, 322.2, 174.1, 139.0; MS-HR (ESI) Calcd for $\mathrm{C}_{39} \mathrm{H}_{43} \mathrm{ClN}_{4} \mathrm{O}_{3}: 650.30237$. Found: 650.30199 . 
$N$-(1,2,3,4-tetrahydro-9-acridinyl)-(8-aminooctyl)2-\{1-[(4-chlorophenyl)carbonyl]-5-methoxy-2-methyl-1H-indol-3-yl\}acetamide hydrochloride (3g)

To solution of $2 \mathrm{~g}(0.20 \mathrm{~g} ; 0.30 \mathrm{mmol})$ in methanol $(1 \mathrm{~mL})$ $1.0 \mathrm{M} \mathrm{HCl}$ in diethyl ether $(4 \mathrm{~mL})$ was added in excess. Yield $83.33 \%$, beige solid, mp $146-148{ }^{\circ} \mathrm{C}$. FTIR (ATR) $\mathrm{v}\left(\mathrm{cm}^{-1}\right): 754.3,1222.6,1319.9,1455.9,1588.0,2926.5$, 3207.1; ${ }^{1} \mathrm{H}$ NMR $\left(600 \mathrm{MHz}\right.$, Methanol- $\left.d_{4}\right) \delta 8.37(1 \mathrm{H}, \mathrm{d}$, $J=8.7 \mathrm{~Hz}, \mathrm{Ar}), 7.86(1 \mathrm{H}, \mathrm{t}, J=7.7 \mathrm{~Hz}, \mathrm{Ar}), 7.76(1 \mathrm{H}, \mathrm{d}$, $J=8.5 \mathrm{~Hz}, \mathrm{Ar}), 7.66-7.70(2 \mathrm{H}, \mathrm{m}, \mathrm{Ar}), 7.55-7.60(3 \mathrm{H}, \mathrm{m}$, $\mathrm{Ar}), 7.04(1 \mathrm{H}, \mathrm{s}, \mathrm{Ar}), 6.91(1 \mathrm{H}, \mathrm{d}, J=9.0 \mathrm{~Hz}, \mathrm{Ar}), 6.64(1 \mathrm{H}$, d, $J=11.5 \mathrm{~Hz}, \mathrm{Ar}), 3.87-3.91\left(2 \mathrm{H}, \mathrm{m}, \mathrm{CH}_{2}\right), 3.80(3 \mathrm{H}, \mathrm{s}$, $\left.\mathrm{CH}_{2}\right), 3.61\left(2 \mathrm{H}, \mathrm{s}, \mathrm{CH}_{2}\right), 3.20-3.24\left(2 \mathrm{H}, \mathrm{m}, \mathrm{CH}_{2}\right), 3.02(2 \mathrm{H}$, $\left.\mathrm{t}, J=5.8 \mathrm{~Hz}, \mathrm{CH}_{2}\right), 2.69(2 \mathrm{H}, \mathrm{t}, J=5.4 \mathrm{~Hz}, 2 \mathrm{H}), 1.93-2.01$ $\left(4 \mathrm{H}, \mathrm{m}, \mathrm{CH}_{2}\right), 1.79\left(2 \mathrm{H}, \mathrm{p}, J=7.6 \mathrm{~Hz}, \mathrm{CH}_{2}\right), 1.51(2 \mathrm{H}, \mathrm{p}$, $\left.J=6.5 \mathrm{~Hz}, \mathrm{CH}_{2}\right), 1.25-1.41\left(8 \mathrm{H}, \mathrm{m}, \mathrm{CH}_{2}\right) ; \mathrm{MS}(\mathrm{ESI}) \mathrm{m} / \mathrm{z}$ $(\mathrm{M}+1)$ 665.3, 336.2, 174.1, 143.0; MS-HR (ESI) Calcd for $\mathrm{C}_{40} \mathrm{H}_{46} \mathrm{ClN}_{4} \mathrm{O}_{3}$ : 664.31802. Found: 664.31826.

\section{$N$-(1,2,3,4-tetrahydro-9-acridinyl)-(9-aminononyl)- 2-\{1-[(4-chlorophenyl)carbonyl]-5-methoxy-2-me- thyl-1H-indol-3-yl\}acetamide hydrochloride (3h)}

To solution of $\mathbf{2 h}(0.20 \mathrm{~g} ; 0.29 \mathrm{mmol})$ in methanol $(1 \mathrm{~mL})$ $1.0 \mathrm{M} \mathrm{HCl}$ in diethyl ether $(4 \mathrm{~mL})$ was added in excess. Yield $56.52 \%$, brown solid, mp $146-148{ }^{\circ} \mathrm{C}$. FTIR (ATR) $\mathrm{v}\left(\mathrm{cm}^{-1}\right): 753.9,1218.7,1397.6,1468.6,1601.7,2916.0$, 3208.0; ${ }^{1} \mathrm{H}$ NMR $\left(600 \mathrm{MHz}\right.$, Methanol- $\left.d_{4}\right) \delta 8.38(1 \mathrm{H}, \mathrm{d}$, $J=8.4 \mathrm{~Hz}, \mathrm{Ar}), 7.86(1 \mathrm{H}, \mathrm{t}, J=7.7 \mathrm{~Hz}, \mathrm{Ar}), 7.76(1 \mathrm{H}, \mathrm{d}$, $J=9.3 \mathrm{~Hz}, \mathrm{Ar}), 7.67-7.71(2 \mathrm{H}, \mathrm{m}, \mathrm{Ar}), 7.55-7.62(3 \mathrm{H}, \mathrm{m}$, Ar), $7.04(1 \mathrm{H}, \mathrm{s}, \mathrm{Ar}), 6.94(1 \mathrm{H}, \mathrm{d}, J=9.0 \mathrm{~Hz}, \mathrm{Ar}), 6.65(1 \mathrm{H}$, d, $J=11.5 \mathrm{~Hz}, \mathrm{Ar}), 3.90-3.94\left(2 \mathrm{H}, \mathrm{m}, \mathrm{CH}_{2}\right), 3.81(3 \mathrm{H}, \mathrm{s}$, $\left.\mathrm{CH}_{2}\right), 3.61\left(2 \mathrm{H}, \mathrm{s}, \mathrm{CH}_{2}\right), 3.20-3.24\left(2 \mathrm{H}, \mathrm{m}, \mathrm{CH}_{2}\right), 3.02(2 \mathrm{H}$, $\left.\mathrm{t}, J=5.9 \mathrm{~Hz}, \mathrm{CH}_{2}\right), 2.70\left(2 \mathrm{H}, \mathrm{t}, J=5.7 \mathrm{~Hz}, \mathrm{CH}_{2}\right), 1.94-2.02$ $\left(4 \mathrm{H}, \mathrm{m}, \mathrm{CH}_{2}\right), 1.80\left(2 \mathrm{H}, \mathrm{p}, J=7.4 \mathrm{~Hz}, \mathrm{CH}_{2}\right), 1.50(2 \mathrm{H}$, $\left.\mathrm{p}, J=6.8 \mathrm{~Hz}, \mathrm{CH}_{2}\right), 1.27-1.43\left(10 \mathrm{H}, \mathrm{m}, \mathrm{CH}_{2}\right) ; \mathrm{MS}$ (ESI) $\mathrm{m} / \mathrm{z}(\mathrm{M}+1) 679.3,174.1,167.0$; MS-HR (ESI) Calcd for $\mathrm{C}_{41} \mathrm{H}_{47} \mathrm{ClN}_{4} \mathrm{O}_{3}: 678.33367$. Found: 678.33207 .

\section{In vitro inhibition studies on $\mathrm{AChE}$ and $\mathrm{BuChE}$}

The inhibitory potency of the de novo synthesized compounds against acetycholinoesterase (AChE from electric ell, EC 3.1.1.7; Sigma-Aldrich) and butyrylcholinesterase (BuChE from equine serum, EC 3.1.1.8; Sigma-Aldrich) was measured using Ellman's method with modifications (Ellman GL, et al. 1961; Czarnecka et al. 2018). The inhibitory activity of the tested compounds was compared with that of the well-known AChE inhibitors tacrine and donepezil. The reactive mixture included: dithiobis-(2-nitro)benzoic acid solution $(0.4 \mathrm{mg} / \mathrm{mL}), \mathrm{AChE}(2 \mathrm{U} / \mathrm{mL})$ or BuChE (4 U/ $\mathrm{mL}$ ) solution and different tested compound concentrations
(0.0001-10 $\mu \mathrm{M})$ (USP XXII-NF XVII United States Pharmacopeia Convention, Inc. 1990). PBS buffer (pH 8.0) was used as a solvent. To start the reaction, acetylthiocholine iodide solution $(1 \mathrm{mM})$ was added. Samples were incubated at room temperature for $10 \mathrm{~min}$ and the change of absorbance was measured at a wavelength of $412 \mathrm{~nm}$ (Synergy H1 spectrophotometer, Bio-Tek). The results were used to calculate $\mathrm{IC}_{50}$ values for each compound. (i.e. the concentration causing 50\% inhibition of enzyme activity). All experiments were carried out in triplicate for each of the three samples.

\section{Kinetic characterization of $\mathrm{AChE}$ and BuChE inhibi- tion}

Ellman's method (on 96-well plates) was used to prepare a kinetic characterization of $\mathrm{AChE}$ and $\mathrm{BuChE}$ inhibition for the tested compound (3h). A mixture of different concentrations of substrate was used: acetylthiocholine iodide (AChI) $(50-350 \mu \mathrm{M})$, four concentrations were used for AChE kinetics $(4,7,14,21 \mathrm{nM})$ and three concentrations for BuChE kinetics $(28,42,56 \mathrm{nM})$, dithiobis-(2-nitro)benzoic acid solution $(0.4 \mathrm{mg} / \mathrm{mL})$, AChE solution $(2 \mathrm{U} / \mathrm{mL})$ or BuChE solution (4 U/mL) (USP XXII-NF XVII United States Pharmacopeia Convention, Inc. 1990). The absorbance at $412 \mathrm{~nm}$ was measured (Synergy H1 Spectrophotometer, Bio-Tek). The obtained results were used to prepare a Lineweaver-Burk plots of $1 /$ velocity versus 1 /substrate concentration. The $K_{\mathrm{m}}$ and $V_{\max }$ values were calculated and the type of enzyme inhibition was determined (Czarnecka et al. 2018).

\section{Cytotoxicity studies on HepG2 cell line}

\section{Cell line}

Cytotoxicity tests were performed using the human hepatocellular liver carcinoma cell line HepG2 (passage 31-38) purchased from Health Protection Agency Culture Collections (ECACC, Salisbury, UK). Eagle's Minimum Essential Medium MEM (Sigma Aldrich, St. Louis, USA) was used for the routine culture of cells. Medium was supplemented with $10 \%$ fetal bovine serum FBS, non-essential amino acid solution (Sigma Aldrich, St. Louis, USA) and 1\% L-Glutamine solution (Sigma Aldrich, St. Louis, USA). The cells were cultured in humidified conditions with $5 \% \mathrm{CO}_{2}$ at $37{ }^{\circ} \mathrm{C}$, the culture medium was discarded every $2-3$ days. During passaging, the cells were briefly rinsed with PBS, aspirated and then incubated for 5-7 min with $1 \mathrm{~mL}$ of $0.05 \%$ trypsin-EDTA solution (Sigma Aldrich, St. Louis, USA) at $37^{\circ} \mathrm{C}$. For the cytotoxicity evaluation, one group of HepG2 cells was exposed to examined compounds and the second one was utilized as a control group. 


\section{Cytotoxicity measurement}

Cytotoxicity test was performed using CellTiter 96 Aqueous One Solution Reagent containing [3-(4,5-dimethylthiazol-2-yl)-5-(3-carboxymethoxyphenyl)-2-(4-sulfophenyl)2H-tetrazolium (MTS), stabilized by phenazine ethosulfate (PSE). MTS is reduced by living cells, thus forming a formazan product, staining the tested probe. The color intensity at $490 \mathrm{~nm}$ is directly proportional to the amount of living cells in the sample (Technical Bulletin 2019). Cells were seeded in a 96-well plate in a density of 10,000 cells per well. After $24 \mathrm{~h}$ of incubation in appropriate conditions, solutions of investigated substances were applied in triplicate (volume of injection $=1 \mu \mathrm{L}$ ). These solutions were prepared by dissolving substances in DMSO and were subsequently diluted to the required range of injection concentrations $(100 ; 50 ; 25 ; 10 ; 5 ; 2.5 ; 1.0 ; 0.5 \mu \mathrm{M})$. Additionally, cells with $100 \%$ viability (injection of $1 \mu \mathrm{L}$ of DMSO into each well), $0 \%$ viability (10 $\mu \mathrm{L}$ of DMSO) and vehicle controls were tested in triplicate.

The cells were incubated at $37{ }^{\circ} \mathrm{C}$ for $24 \mathrm{~h}$ in an atmosphere containing $5 \% \mathrm{CO}_{2}$ and then $20 \mu \mathrm{L}$ of the reagent from the kit CellTiter 96 Aqueous One Solution Cell Proliferation Assay (PROMEGA, Fitchburg, USA) were applied into each well. After $80 \mathrm{~min}$ of incubation, absorbance was measured at $490 \mathrm{~nm}$ (TECAN, Infinite M200 Austria). IC $_{50}$ was calculated using nonlinear regression from a semilogarithmic plot of incubation concentration versus the percentage of absorbance relative to control cells. Statistical parameters (means and standard deviations) were obtained utilizing GraphPad Prism software (version 6).

\section{Cytotoxicity studies on EA.hy926 cell line}

\section{Cell culture}

The EA.hy926 cell line (the human umbilical vein, somatic cell hybrid) (American Type Culture Collection) was used to evaluate the cytotoxicity after $3 \mathrm{~h}$. The culture medium consisted of Dulbecco's Modified Eagle's Medium (DMEM) (PAN-Biotech) containing 10\% Fetal Bovine Serum (Sigma Aldrich), $2 \mathrm{mM}$ Glutamine (Sigma Aldrich) and 100 units/ $\mathrm{mL}$ penicillin and $100 \mathrm{mg} / \mathrm{mL}$ streptomycin (Biological Industries). Cells were passaged and kept in an incubator at $37{ }^{\circ} \mathrm{C}$ with $5 \% \mathrm{CO}_{2}$ before the initiation of the assay.

\section{MTT cell viability assay}

The MTT test was performed to evaluate cell metabolic activity and to determine cell viability. Enzymatically active mitochondria reduce the water-soluble MTT (3-(4,5-dimethylthiazol-2-yl)-2,5 diphenyltetrazoliumbromide) to blue-violet insoluble formazan crystals. At the first day of assay, cells were seeded at a density of 104 cells/well in 96-well plates and incubated for $24 \mathrm{~h}$ at $37{ }^{\circ} \mathrm{C}$ and $5 \% \mathrm{CO}_{2}$. After this time, culture medium from all wells was removed and replaced by $100 \mu \mathrm{L}$ of the compound solution over the range

of concentration $(35-1 \mu \mathrm{M})$ or by $100 \mu \mathrm{L}$ of culture medium (blank control). Plates were incubated for $24 \mathrm{~h}$ at $37^{\circ} \mathrm{C}$ and $5 \% \mathrm{CO}_{2}$. Finally, the culture medium and compound solutions were removed from all wells and replaced by $50 \mu \mathrm{L}$ of the MTT solution $(0.75 \mathrm{mg} / \mathrm{mL})$ and incubated in the dark for $2 \mathrm{~h}$ at $37{ }^{\circ} \mathrm{C}$ and $5 \% \mathrm{CO}_{2}$. Then the MTT solution was carefully removed and $100 \mu \mathrm{L}$ of DMSO (Serva) was added to each well to dissolve the formazan crystals. The plates were incubated for $10 \mathrm{~min}$ at room temperature.

After incubation, $5 \mu \mathrm{L}$ of Sorenson's glycine buffer (0.1 M glycine, $0.1 \mathrm{M} \mathrm{NaCl}, \mathrm{pH} 10.5)$ was added to each well. Plates were incubated at room temperature for $5 \mathrm{~min}$. Then the absorbance was measured in the microplate reader (Synergy H1, BioTek, Winooski, VT, USA) at $570 \mathrm{~nm}$. Cell viability was expressed as a percentage of control values (blank) (Plumb 1999).

\section{pKa assay}

Potassium dihydrogen phosphate, potassium hydroxide, methanol (POCH) solution was used to prepare a buffer solution. Stock phosphorous buffer was prepared from $500 \mathrm{~mL}$ $0.02 \mathrm{M}$ potassium dihydrogen phosphate solution by adding $500 \mathrm{~mL}$ methanol. 35 work buffers were prepared by titrating stock phosphorus buffer by $0.1 \mathrm{M}$ potassium hydroxide solution in methanol: water $(1: 1)$ mix. Work buffer $\mathrm{pH}$ was set in the range from 5.6 to 12.4 by 0.2 per step. The $\mathrm{pH}$ measurement was performed at $23^{\circ} \mathrm{C}$ using Mettler Toledo FiveEasy pH-meter with an LE438 Lab pH electrode (Mettler Toledo). HI7004 pH 4.01 buffer solution and HI7007 pH 7.01 buffer solution (Hanna instruments Inc., Woodsocket RI, USA) were used for the calibration of the $\mathrm{pH}$ meter. Our tested compound solution was $5 \mu \mathrm{M}$ solution in methanol: water (1:1) mix.

Spectrophotometric measurement was performed in a 96-well plate using Synergy H1 microplate reader (BioTek) with Gen5 software (BioTek). The full assay consisted of 35 UV spectra measurements, one for each working buffer solution. For the assay, $180 \mu \mathrm{L}$ sequent working buffer and $20 \mu \mathrm{L}$ tested compound solution was added to each of 35 wells. For the blank, $200 \mu \mathrm{L}$ sequent working buffer only was added to every 35 wells. The measurement was performed at $23{ }^{\circ} \mathrm{C}$, from $280 \mathrm{~nm}$ to $380 \mathrm{~nm}$ in $1 \mathrm{~nm}$ steps. The obtained spectra were subtracted from the blank. Results collected at $310 \mathrm{~nm}$ and $336 \mathrm{~nm}$ were selected for further analysis. All calculations were performed based on $310 / 336 \mathrm{~nm}$ and $336 / 310 \mathrm{~nm}$ ratios. The specific $\mathrm{pH}$ value for every ionized form of the molecule was estimated by finding the smallest difference between the absorbance ratios of $310 / 336 \mathrm{~nm}$ 
and $336 / 310 \mathrm{~nm}$. The pKa value was calculated for both ratios. The mean of these two results was considered as the final pKa value. The obtained results were compared with computer calculations of pKa carried out by online software chemicalize.com (ChemAxon 2018) (Musil et al. 2016).

\section{In vitro $A B T S$ and DPPH assay}

The antioxidant activity of the synthesized compounds $(\mathbf{3 a}-\mathbf{3 h})$ was evaluated based on two different assays: radical scavenging activities using 2,2-diphenyl-1-picrylhydrazyl (DPPH) assay and ABTS assay. In the ABTS assay, $5 \mathrm{~mL}$ of a $7 \mathrm{mM}$ aqueous ABTS solution was mixed with $88 \mu \mathrm{L}$ of a $140 \mathrm{mM}$ (2.45 mM final concentration) potassium persulfate $\left(\mathrm{K}_{2} \mathrm{~S}_{2} \mathrm{O}_{8}\right)$. The mixture was stored at room temperature for $16 \mathrm{~h}$. After this time, $\mathrm{ABTS}^{\bullet+}$ was diluted in methanol; absorbance at $734 \mathrm{~nm}$ was found to be $0.7 \pm 0.02$. Solutions of each tested compound were dissolved in methanol. The decrease in absorbance was recorded at 0 and after 6 min (Re et al. 1999; Nenadis et al. 2007).

In the DPPH assay, $50 \mu \mathrm{L}$ of various concentrations of tested compounds were mixed with an equal quantity of DPPH solution $(0.2 \mathrm{mM})$. After $30 \mathrm{~min}$ of incubation in the dark, the absorbance was measured at $517 \mathrm{~nm}$. Compounds with antioxidant activity reduce the color

of the DPPH solution (Blois 1958; Chand et al. 2016). Both methods were carried out using a Synergy H1 96-well microplate (Biotek). All measurements were carried out in triplicate with blank solutions each time and compared to the references. Compounds concentration providing 50\% inhibition $\left(\mathrm{FRS}_{50}\right)$ was calculated from the graph plotted as inhibition percentage against compound concentration.

\section{Molecular modeling}

Docking studies were performed with GoldSuite 5.1 (CCDC) (Lu et al. 2014). Three-dimentional structure of all tested ligands was prepared with Corina on-line (Molecular Networks and Altamira) (Mailloux et al. 2001; Corina on-line 2019). Sybyl X-1.1 (Tripos) was used to check atom types, add hydrogen atoms and assign Gasteiger-Marsili charges (Gasteiger and Marsili 1978; Sybyl-X 1.12010 ). Due to the high conformational freedom of the amino acids needed for the ligand binding in the active site of acetylcholinesterase (Phe330 and Trp279), prepared ligands were docked to three structures of acetylcholinesterase (PDB codes:1EVE, $1 \mathrm{ACJ}$ and $2 \mathrm{CKM})$. The acetylcholinesterase complex with bis-(7)-tacrine (PDB code: 2CKM) was chosen as the most suitable for docking as it gave the most consistent results in preliminary studies. The butyrylcholinesterase complex with butyrate (PDB code: 1P0I) was chosen for docking studies on this enzyme. Protein structures were prepared with Hermes 1.7.0. to protonate all histidine residues in
$\mathrm{N} \varepsilon$ position, add all hydrogen atoms, remove unnecessary ligands and water molecules. Docking was performed into binding sites composed of all amino acids within radius of $10 \AA$ from bis-(7)-tacrine for AChE and $20 \AA$ from the glycerol molecule present in the active center of BuChE. Standard settings of the genetic algorithm were applied. Ten poses per ligand, sorted by GoldScore (for AChE) and ChemScore (for BChE) function values were obtained. PyMOL 0.99rc6 (DeLano Scientific LLC) was used to visualize the results of docking (Bajda et al. 2015; PyMOL 0.99rc6 2006).

\section{Results and discussion}

\section{Synthesis}

The new acetylcholinesterase inhibitors were created by connecting diamine derivatives of tetrahydroacridine with indomethacin. Compounds 1a-1h (synthesis described by Szymanski et al. 2011) were connected to an acid molecule by aliphatic linker (Scheme 1). Reactions were carried out in the presence of 2-chloro-4,6-dimethyl-1,3,5-triazine (CDMT) and $N$-methylmorpholine. CDMT was necessary for the activation of the carboxylic group in indomethacin. CDMT, indomethacin and $N$-methylmorpholine were dissolved in tetrahydrofuran (THF). Next, amines $\mathbf{1 a - 1 h}$ were added. After $24 \mathrm{~h}$ at $-5{ }^{\circ} \mathrm{C}$ products $\mathbf{2 a}-2 \mathrm{~h}$ were obtained. The reactions were monitored by TLC. Compounds $\mathbf{2 a}-\mathbf{2 h}$ were purified by flash chromatography using a PuriFlash 430 (Interchem Inc). In the last stage, $\mathbf{2 a - 2 h}$ were converted to 3a-3h hydrochlorides. They were dissolved in methanol and $\mathrm{HCl}$ in ether was added (room temp., $24 \mathrm{~h}$ ). Hydrochlorides 3a-3h were crystallized.

\section{In vitro inhibition studies on $\mathrm{AChE}$ and BuChE}

\section{The inhibitory activity against $\mathrm{AChE}$ and $\mathrm{BuChE}$ for de novo synthesized compounds}

3a-3h was measured using Ellman's method (Ellman et al. 1961; Czarnecka et al. 2018). The activities of the tested compounds were compared with tacrine and donepezil (well-known cholinesterase inhibitors). $\mathrm{IC}_{50}$ values for tested compound are shown in Table 1. Compounds $\mathbf{3 b}-\mathbf{3 h}$ exhibit high inhibitory activity against $\mathrm{AChE}$. The $\mathrm{IC}_{50}$ values for $\mathbf{3 b} \mathbf{b} \mathbf{3 h}$ ranging from 10 to $260 \mathrm{nM}$. Compound $\mathbf{3 a}$ has lower inhibitory potency than others, with an $\mathrm{IC}_{50}$ value of $7078 \mathrm{nM}$. In comparison to tacrine $\left(\mathrm{IC}_{50}=174 \mathrm{nM}\right), \mathbf{3 b}-\mathbf{3 d}$ and $\mathbf{3 f}-\mathbf{3 h}$ revealed higher inhibitory activity. Compared to donepezil $\left(\mathrm{IC}_{50}=87 \mathrm{nM}\right), \mathbf{3 b}-\mathbf{3 d}$ and $\mathbf{3 g}-\mathbf{3 h}$ revealed higher inhibition. The most active compound against $\mathrm{AChE}$ was $\mathbf{3 h}$ $\left(\mathrm{IC}_{50}=10 \mathrm{nM}\right.$ ), with the longest alkyl linker (nine carbon atoms). 
Table 1 The activity of tested compounds against AChE and $\mathrm{BuChE}$

\begin{tabular}{lcccc}
\hline Compound & $\mathrm{AChE} \mathrm{IC}_{50} \pm \mathrm{SD}^{\mathrm{A}}(\mathrm{nM})$ & $\begin{array}{l}\mathrm{BuChE} \mathrm{IC}_{50} \pm \mathrm{SD}^{\mathrm{B}} \\
(\mathrm{nM})\end{array}$ & $\begin{array}{l}\text { Selectivity for } \\
\mathrm{AChE}^{\mathrm{C}}\end{array}$ & $\begin{array}{l}\text { Selectivity } \\
\text { for BChE }\end{array}$ \\
\hline $3 \mathrm{a}$ & $7086 \pm 1355$ & $289 \pm 36$ & 0.04 & 24.56 \\
$3 \mathrm{~b}$ & $42 \pm 4$ & $335 \pm 33$ & 8.05 & 0.12 \\
$3 \mathrm{c}$ & $25 \pm 2$ & $229 \pm 30$ & 9.16 & 0.11 \\
$3 \mathrm{~d}$ & $62 \pm 8$ & $30 \pm 4$ & 0.49 & 2.06 \\
$3 \mathrm{e}$ & $261 \pm 6$ & $246 \pm 26$ & 0.94 & 1.06 \\
$3 \mathrm{f}$ & $99 \pm 3$ & $7 \pm 1$ & 0.07 & 15.25 \\
$3 \mathrm{~g}$ & $29 \pm 2$ & $241 \pm 44$ & 8.40 & 0.12 \\
$3 \mathrm{~h}$ & $10 \pm 1$ & $57 \pm 4$ & 6.02 & 0.17 \\
Tacrine & $174 \pm 31$ & $32 \pm 5$ & 0.19 & 5.40 \\
Donepezil & $87 \pm 15$ & $9757 \pm 1113$ & 112.17 & 0.01 \\
\hline
\end{tabular}

${ }^{\mathrm{A}}$ Compound concentration (mean $\pm \mathrm{SD}$ of the three independent experiments) for $50 \%$ inactivation of AChE

${ }^{\mathrm{B}}$ Compound concentration (mean $\pm \mathrm{SD}$ of the three independent experiments) for $50 \%$ inactivation of $\mathrm{BuChE}$

${ }^{\mathrm{C}} \mathrm{IC}_{50}(\mathrm{BuChE}) / \mathrm{IC}_{50}(\mathrm{AChE})$

${ }^{\mathrm{D}} \mathrm{IC}_{50}(\mathrm{AChE}) / \mathrm{IC}_{50}(\mathrm{BuChE})$
Compounds $\mathbf{3 d}\left(\mathrm{IC}_{50}=30 \mathrm{nM}\right)$ and $\mathbf{3 f}\left(\mathrm{IC}_{50}=6 \mathrm{nM}\right)$ were more active against $\mathrm{BuChE}$ than tacrine $\left(\mathrm{IC}_{50}=32 \mathrm{nM}\right)$. All of the compounds with $\mathrm{IC}_{50}$ from 6 to $335 \mathrm{nM}$ revealed a higher inhibitory potency against BuChE than donepezil $\left(\mathrm{IC}_{50}=9757 \mathrm{nM}\right)$.

Compound $3 \mathbf{h}$ was more active against $\mathrm{AChE}$ than tacrine (18 times more) and donepezil (9 times more) and was selected for kinetic studies of the AChE inhibition. Compound $\mathbf{3 f}$ was more active against $\mathrm{BuChE}$ than tacrine (5 times more) and donepezil (1501 times more) and was selected for kinetic studies of BuChE inhibition.

\section{Kinetic characterization of $\mathrm{AChE}$ and $\mathrm{BChE}$ inhibition}

The study of the inhibitory mechanism against AChE showed $\mathbf{3 h}$ to have the highest activity $\left(\mathrm{IC}_{50}=10 \mathrm{nM}\right)$. The activity of enzymes at various substrate concentrations was measured. The Lineweaver-Burk plot of reciprocal velocity versus reciprocal substrate concentrations for the various inhibitor concentrations was created. The $K_{\mathrm{m}}$ values decreased, and $V_{\max }$ values increase at increasing inhibitor concentrations.

Compound $\mathbf{3 h}$ demonstrated mixed-type inhibition against $\mathrm{AChE}$ and BuChE (Figs. 1 and 2) (Czarnecka et al. 2018).

\section{Cytotoxicity studies on HepG2 cell line}

To compare the cytotoxicity of investigated substances, $\mathrm{IC}_{50}$ was calculated: the inhibitory concentration which reduces the viability of the cells' population to $50 \%$ from the control viability $(100 \%)$. Derivatives of 2-(1-(4-chlorobenzoyl)-5-methoxy-2-methyl-1 $H$-indol-3-yl)-

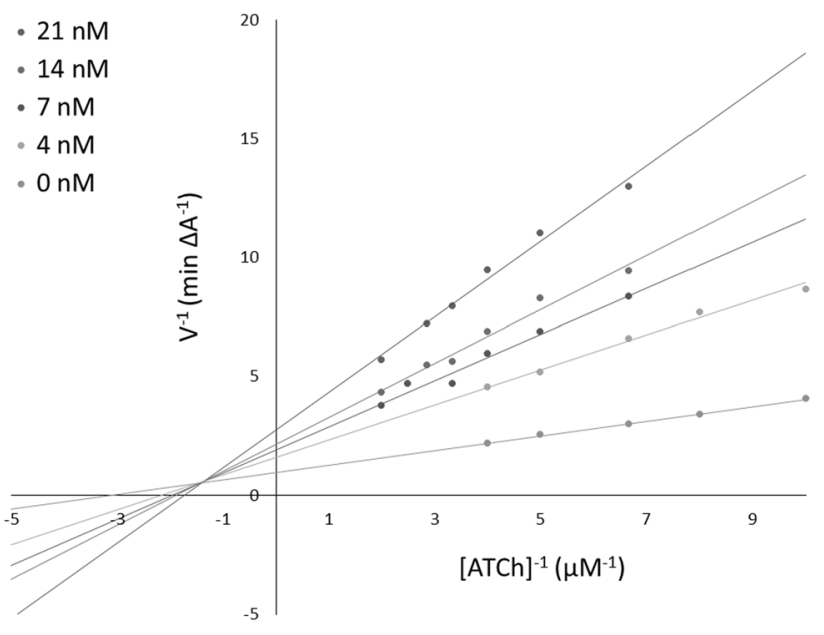

Fig. 1 Lineweaver-Burk plot showing a mixed-type AChE inhibition for $3 h$

$N$-( $\omega$-(5,6,7,8tetrahydroacridin-9-ylamino)ethyl) acetamide hydro-chlorides values of $\mathrm{IC}_{50}$ were relatively uniform and vary from $5 \mu \mathrm{M}$ (compound 3d) to $17 \mu \mathrm{M}$ (compound 3c).

\section{Cytotoxicity studies on EA.hy926 cell line}

The EA.hy926 cells are immortalized human vein endothelial cells (HUVEC), which show morphological, phenotypic and functional characteristics of human endothelial cells, without the limited lifespan. They are considered a suitable model for drug screening ( $\mathrm{Lu}$ et al. 2014). The endothelium is a barrier between blood and tissues, controlling coagulation and the adhesion of immunocompetent 


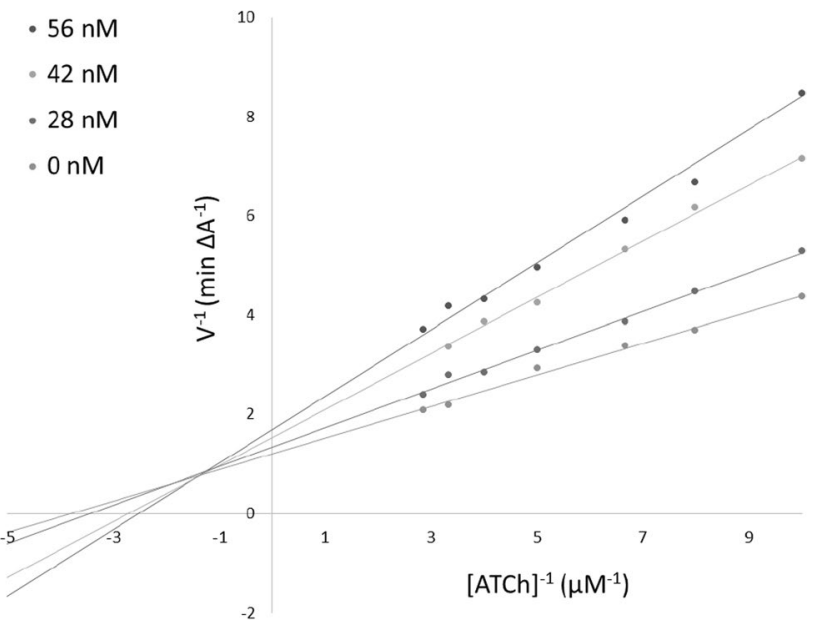

Fig. 2 Lineweaver-Burk plot showing a mixed-type inhibition for $\mathrm{BuChE}$ (3h)

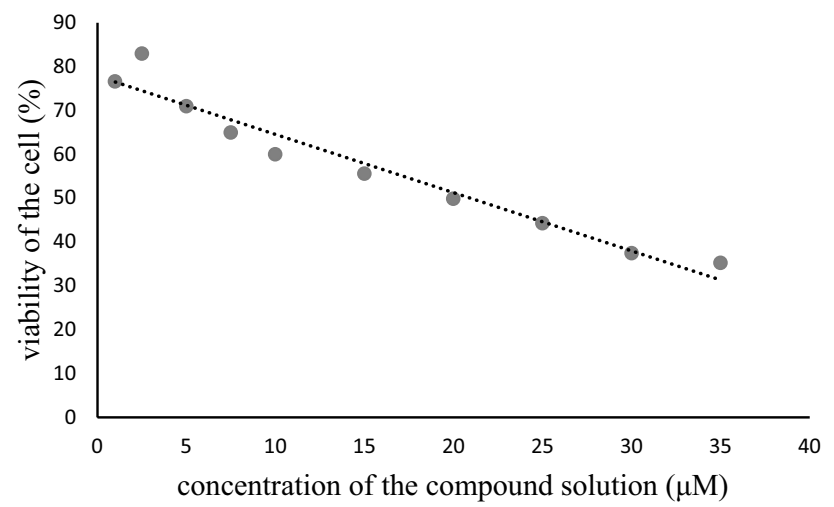

Fig. 3 Percentage of EA.hy926 cell viability relative to different concentrations of $\mathbf{3 h}$ cells, and modulating the vascular tone. Many chemical agents or chronic inflammatory diseases injure the vascular endothelium.

Due to injury, the endothelium might lose its integrity leading to cell necrosis or apoptosis (Mailloux et al. 2001). The cytotoxicity of $\mathbf{3 h}$ was investigated on EA.hy926 cell line. The $\mathrm{IC}_{50}$ value was of $20 \pm 2 \mu \mathrm{M}$. It might be concluded that $\mathbf{3 h}$ was slightly less toxic against EA.hy926 cells than on HepG2 cells and did not cause strong injury of endothelial cells (Fig. 3).

\section{pKa assay}

$\mathrm{pKa}$ is an important physical property, especially for the drug industry. This study used the methodology developed by Musil et al. (2016). Our spectrophotometric screening method allowed us to determine two $\mathrm{pKa}$ values of compound $3 \mathrm{~h}$ within the $\mathrm{pH}$ range from 5.6 to 12.4 . The change of UV spectra is presented in Fig. 4. Both pKa values were estimated experimentally and by modelling to estimate the specific $\mathrm{pH}$ value for each ionized form (Fig. 5). pKa was calculated by regression. The experimental $\mathrm{pKa}_{1}$ value is close to the strongest basic pKa value calculated by ChemAxon software; however, a large difference was observed for $\mathrm{pKa}_{2}$ (Table 2).

\section{In vitro ABTS and DPPH assay}

To prove the antioxidant activities of new hybrids (3a-3h), DPPH and ABTS assays were used (Table 3). Trolox was selected as a positive control. Tacrine and indomethacin served as negative standards as they show negligible radical-capturing abilities. Surprisingly, the combination of
Fig. 4 UV spectra of compound 3h at different $\mathrm{pH}$

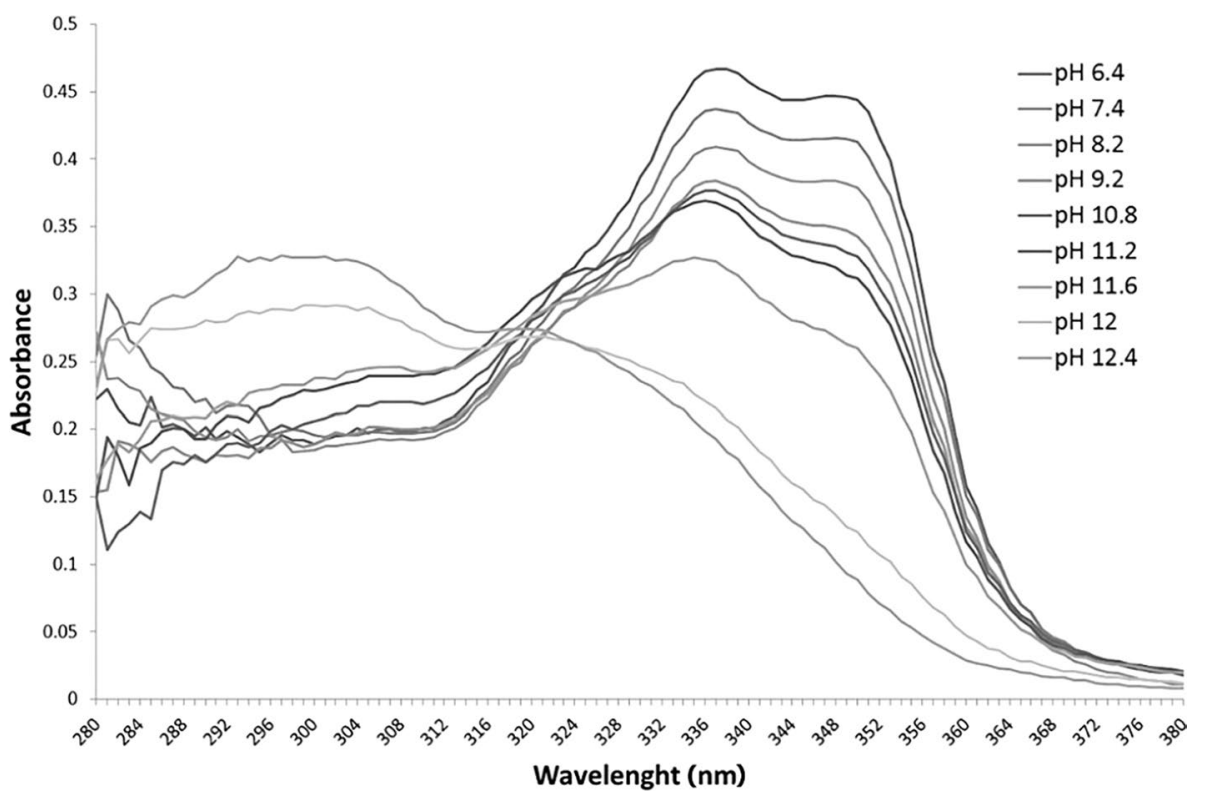


Fig. 5 Scatter plots of obtained for compound $\mathbf{3 h}$ with regression equation
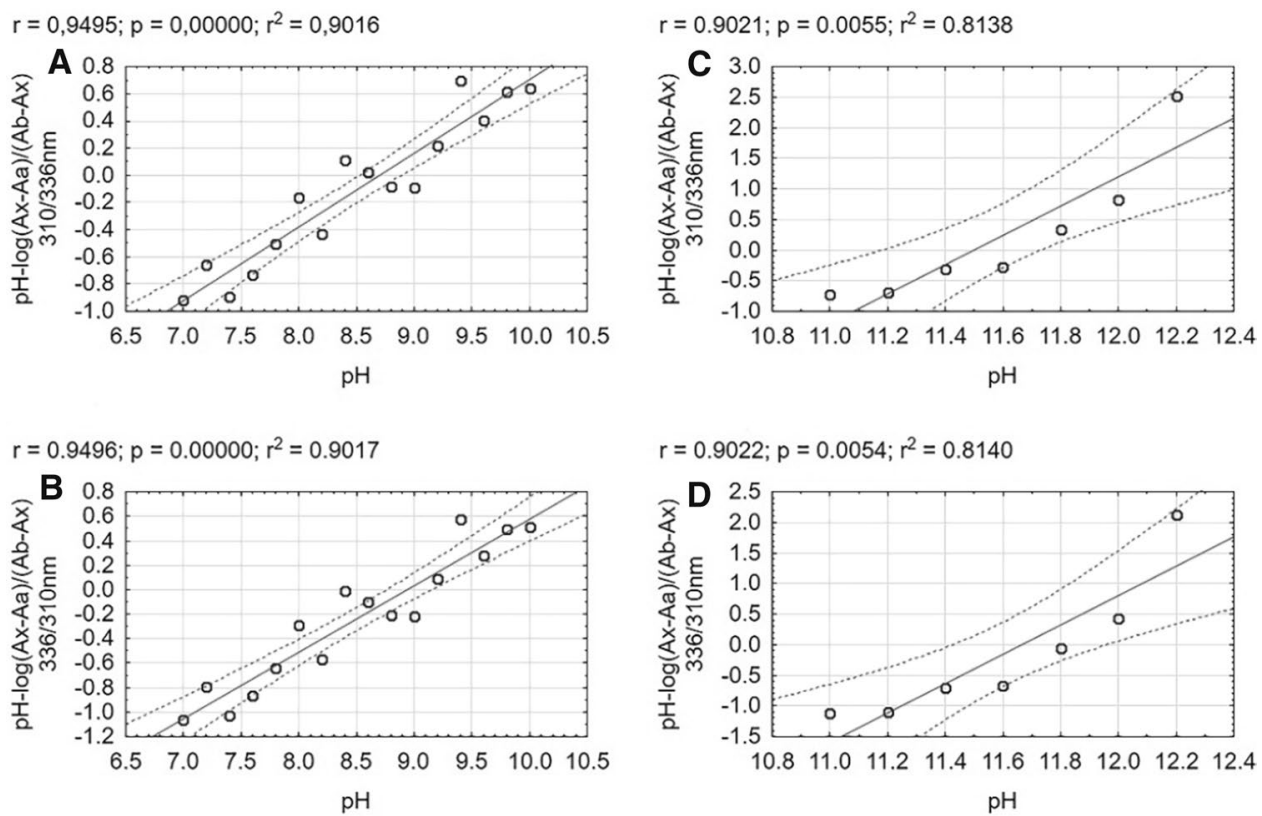

Table 2 Experimental and computer calculated pKa values of compound $\mathbf{3 h}$

\begin{tabular}{llllllll}
\hline $\mathrm{pKa}_{1} 310 / 336 \mathrm{~nm}$ & $\mathrm{pKa}_{1} 336 / 310 \mathrm{~nm}$ & $\mathrm{pKa}_{1}$ & $\mathrm{pKa}_{2} 310 / 336 \mathrm{~nm}$ & $\mathrm{pKa}_{2} 336 / 310 \mathrm{~nm}$ & $\mathrm{pKa}_{2}$ & $\begin{array}{l}\mathrm{pKa} \text { strong basic Che- } \\
\text { mAxon }\end{array}$ & $\begin{array}{l}\mathrm{pKa} \text { strong acidic } \\
\text { ChemAxon }\end{array}$ \\
\hline 8.94 & 8.70 & 8.82 & 11.67 & 11.50 & 11.58 & 8.89 & 15.59 \\
\hline
\end{tabular}

Table 3 In vitro DPPH and ABTS assay results

\begin{tabular}{lll}
\hline Compound & ABTS $($ FRS50, mM) & DPPH $(\text { FRS50, mM })^{\mathrm{a}}$ \\
\hline $3 \mathrm{a}$ & $3.96 \pm 0.17$ & $>10$ \\
$3 \mathrm{~b}$ & $2.36 \pm 0.49$ & $>10$ \\
$3 \mathrm{c}$ & $3.85 \pm 0.61$ & $>10$ \\
$3 \mathrm{~d}$ & $1.34 \pm 0.13$ & $>10$ \\
$3 \mathrm{e}$ & $>10$ & $>10$ \\
$3 \mathrm{f}$ & $0.89 \pm 0.11$ & $5.03 \pm 0.67$ \\
$3 \mathrm{~g}$ & $1.41 \pm 0.14$ & $3.62 \pm 0.46$ \\
$3 \mathrm{~h}$ & $0.51 \pm 0.04$ & $0.60 \pm 0.12$ \\
Tacrine & $>10$ & $>10$ \\
Indomethacine & $>10$ & $>10$ \\
Trolox & $4.2 \times 10^{-2} \pm 0.4 \times 10^{-2}$ & $4.86 \times 10^{-3} \pm 0.42 \times 10^{-3}$ \\
\hline
\end{tabular}

${ }^{\mathrm{a}}$ Each value is the mean of triplicate determinations $\pm \mathrm{SD}$

tacrine and indomethacin with an aliphatic chain improved antioxidant activity. Generally, compounds with longer linkers show better activity. Both studies confirmed that the most active compound was $\mathbf{3 h}$ with nine carbon atoms in the chain, as confirmed by Ellman.

\section{Molecular modeling}

Molecular modeling studies were performed to explain how the tested compounds interacted with cholinesterases. All derivatives demonstrated one predominant binding mode with AChE, similar to the one of bis-(7)-tacrine in the crystal structure (PDB code: 2CKM). The most active derivative (3h) is shown in Fig. 6. The tacrine fragment was located in the catalytic active site, creating characteristic $\pi-\pi$ stacking and cation $-\pi$ interactions with Trp84 and Phe330 and forming a hydrogen bond between the protonated nitrogen atom and oxygen atom from carbonyl group of His440. The linker was directed toward the active site entrance where a fragment of indomethacin was involved in aromatic interactions, especially with Trp279 from peripheral anionic site (PAS). The length of the linker had a direct impact on how indomethacin interacted with the PAS. This fragment in ligands with a shorter linker (3b-3d) created $\pi-\pi$ stacking with Tyr70 and Trp279, similarly to the second tacrine moiety of bis(7)-tacrine. Optimal interaction was ensured if four carbon atoms were present in the linker (3c). Longer alkyl chains (compounds $\mathbf{3 e}-\mathbf{3 h}$ ) led to the lack of interaction of indomethacin with the Tyr70. However, two longest linkers (derivatives $\mathbf{3 g}$ and $\mathbf{3 h}$ ) allowed for the formation 
Fig. 6 Binding mode of compound $\mathbf{3 h}$ (orange sticks) in the active site of AChE. Parts of the active site elements are color-coded: yellow, catalytic triad; magenta, anionic site; orange, acyl pocket; cyan, oxyanion hole; green, PAS. The box depicts a detailed view of binding site with compound $\mathbf{3 h}$

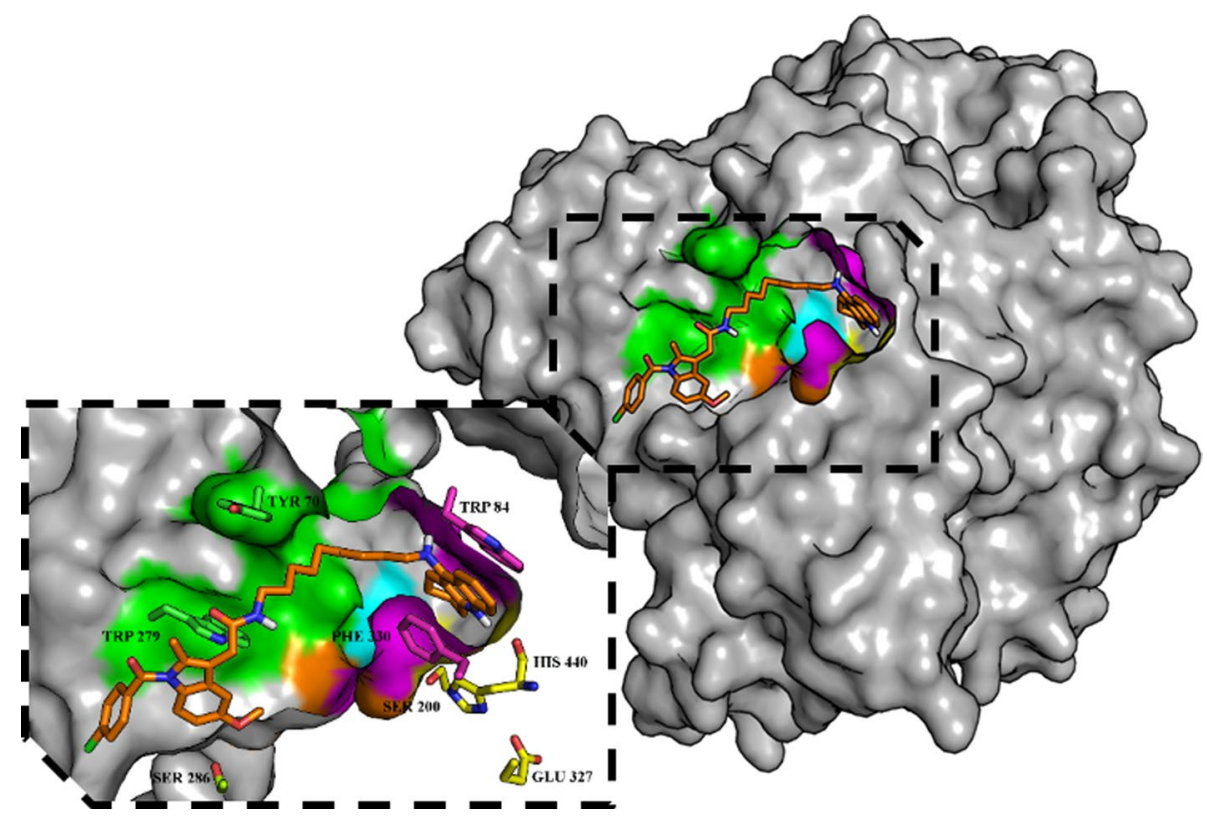

of specific interaction with Ser286 which increased the strength of binding of these ligands with the AChE.

In spite of the fact that the active site of butyrylcholinesterase is wider than the active site of acetylcholinesterase, final conformations of ligands were similar. The most active compound $\mathbf{3 f}$ was used to illustrate the binding mode (Fig. 7). The tacrine moiety presented the same conformation as before. It created $\pi-\pi$ stacking and cation $-\pi$ interactions with $\operatorname{Trp} 82$ and H-bond between protonated nitrogen and carbonyl group of His438. The indomethacin moiety enforced extended conformation for all derivatives despite differences in the length of the linker. Ligands with a shorter linker interacted with reduced PAS of butyrylcholinesterase, while derivatives with a longer chain reached farther and bound to Phe278. The optimal length of the linker (seven carbon atoms for compound $\mathbf{3 f}$ ) allowed for optimal aromatic interactions between indomethacin and Phe278. Changing the length of the linker resulted in the weakening or loss of these interactions, and a consequent decrease in activity.

\section{Conclusions}

The development of drugs that could modulate multiple targets simultaneously in the multifactorial processes involved in Alzheimer's disease is a very good direction for obtaining effective drugs. In these studies, we present the synthesis and evaluation of de novo synthesized hybrids formed from the connection of 9-amino-1,2,3,4-tetrahydroacridine and indomethacin. As a link, different alkyl chains were used.
Fig. 7 Binding mode of compound 3f (orange sticks) in the active site of BuChE. Parts of active site are colorcoded: yellow, catalytic triad; magenta, anionic site; orange, acyl pocket; cyan, oxyanion hole; green, PAS. The box depicts a detailed view of the binding site with compound $\mathbf{3 f}$

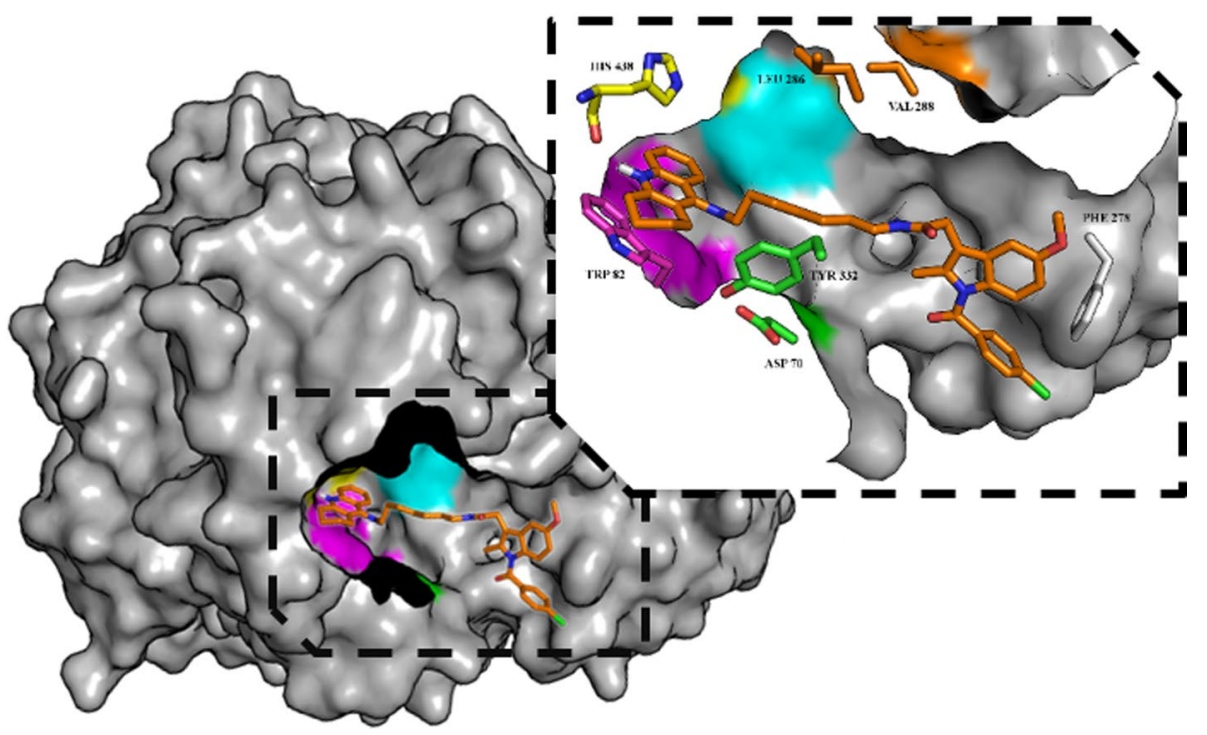


The inhibitory potencies against $\mathrm{AChE}$ and BuChE of received compounds were determined. Results were compared with the activity of well-known cholinesterase inhibitors (tacrine, donepezil). The results show high inhibitory potency of tested compounds, with $\mathrm{IC}_{50}$ in low micromolar and submicromolar range $(7.086 \mu \mathrm{M}$ and $0.009 \mu \mathrm{M})$. Most of them have a higher potency than the reference compounds. For the most active compounds kinetic studies

were performed. Mix-type AChE and BChE inhibition by $\mathbf{3 h}$ was determined by spectrophotometry. In addition, the new compounds exerted low toxicity on the tested cells (HepG2 and EA.hy926), indicating that $\mathbf{3 h}$ could be safer than tacrine but with good bioavailability. Moreover, the compounds showed antioxidant effects in DDPH and ABTS studies. New compounds have many features that can be useful in the fight against Alzheimer's disease: they act simultaneously on several targets, they show lower toxicity than the reference compound-tacrine. The most promising compound (3h) can be used to further research its usefulness as a potential drug. Our results and those of Zhi-Qiang Cheng et al., indicate that indole derivatives can be promising in the formation of hybrids with tacrine in the search for new drugs for Alzheimer's disease. Such hybrids show a significant reduction in hepatotoxicity compared to tacrine and stronger inhibition of cholinesterases.

Acknowledgements Financial support by Grant 2015/19/B/NZ7/02847 from the National Science Centre, Poland is gratefully acknowledged. The research was carried out using the equipment purchased for the funds granted for the project "The equipment of innovative laboratories doing research on new medicines used in the therapy of civilization and neoplastic diseases" within the Operational Program Development of Eastern Poland 2007-2013, Priority Axis I Modern Economy, Operations I.3 Innovation Promotion. Molecular modeling studies were financially supported by the Jagiellonian University Medical College grant no. K/DSC/002860. This work was supported by Charles University (Grants SVV 260414 and PROGRES Q42).

Open Access This article is licensed under a Creative Commons Attribution 4.0 International License, which permits use, sharing, adaptation, distribution and reproduction in any medium or format, as long as you give appropriate credit to the original author(s) and the source, provide a link to the Creative Commons licence, and indicate if changes were made. The images or other third party material in this article are included in the article's Creative Commons licence, unless indicated otherwise in a credit line to the material. If material is not included in the article's Creative Commons licence and your intended use is not permitted by statutory regulation or exceeds the permitted use, you will need to obtain permission directly from the copyright holder. To view a copy of this licence, visit http://creativecommons.org/licenses/by/4.0/.

\section{References}

Allgaier M, Allgaier C (2014) An update on drug treatment options of Alzheimer's disease. Front Biosci (Landmark Ed) 19:1345-1354. https://doi.org/10.2741/4285
Bajda M, Jończyk J, Malawska B, Czarnecka K, Girek M, Olszewska P, Sikora J, Mikiciuk-Olasik E, Skibiński R, Gumieniczek A, Szymański P (2015) Synthesis, biological evaluation and molecular modeling of new tetrahydroacridine derivatives as potential multifunctional agents for the treatment of Alzheimer's disease. Bioorg Med Chem 23(17):5610-5618. https://doi.org/10.1016/j. bmc.2015.07.029

Berk M, Dean O, Drexhage H, McNeil JJ, Moylan S, O’Neil A, Davey CG, Sanna L, Maes M (2013) Aspirin: a review of its neurobiological properties and therapeutic potential for mental illness. BMC Med 11(1):74. https://doi.org/10.1186/1741-7015-11-74

Blass JP (2002) Alzheimer's disease and Alzheimer's dementia: distinct but overlapping entities. Neurobiol Aging 23(6):1077-1084. https://doi.org/10.1016/S0197-4580(02)00036-2

Blois MS (1958) Antioxidant determinations by the use of a stable free radical. Nature 181:1199. https://doi.org/10.1038/1811199a0

Carreras I, McKee AC, Choi JK, Aytan N, Kowall NW, Jenkins BG, Dedeoglu A (2013) R-flurbiprofen improves tau, but not $\mathrm{A} ß$ pathology in a triple transgenic model of Alzheimer's disease. Brain Res 1541:115-127. https://doi.org/10.1016/j.brain res.2013.10.025

Cataldo JK, Prochaska JJ, Glantz SA (2010) Cigarette smoking is a risk factor for Alzheimer's Disease: an analysis controlling for tobacco industry affiliation. J Alzheimers Dis 19(2):465-480. https://doi. org/10.3233/JAD-2010-1240

Chand K, Alsoghier HM, Chaves S, Santos MA (2016) Tacrine(hydroxybenzoyl-pyridone) hybrids as potential multifunctional anti-Alzheimer's agents: AChE inhibition, antioxidant activity and metal chelating capacity. J Inorg Biochem 163:266-277. https:// doi.org/10.1016/j.jinorgbio.2016.05.005

Chen SY, Chen Y, Li YP, Chen SH, Tan JH, Ou TM, Gu LQ, Huang ZS (2011) Design, synthesis, and biological evaluation of curcumin analogues as multifunctional agents for the treatment of Alzheimer's disease. Bioorg Med Chem 19:5596-5604. https:// doi.org/10.1016/j.bmc.2011.07.033

Chen Y, Sun J, Huang Z, Liao H, Peng S, Lehmann J, Zhang Y (2013a) Design, synthesis and evaluation of tacrine-flurbiprofen-nitrate trihybrids as novel anti-Alzheimer's disease agents. Bioorg Med Chem 21(9):2462-2470. https://doi.org/10.1016/j. bmc.2013.03.005

Chen Y, Sun J, Peng S, Liao H, Zhang Y, Lehmann J (2013b) Tacrineflurbiprofen hybrids as multifunctional drug candidates for the treatment of alzheimers disease. Arch Pharm (Weinheim Ger) 346(12):865-871. https://doi.org/10.1002/ardp.201300074

Cheng ZQ, Zhu KK, Zhang J, Song JL, Muehlmann LA, Jiang CS, Liu CL, Zhang H (2019) Molecular-docing-guided design and synthesis of new IAA-tacrine hybrids as multifunctional AChE/BChE inhibitors. Bioorg Chem 83:277-288. https://doi.org/10.1016/j. bioorg.2018.10.057

Corina on-line (2019) http://www.molecular-networks.com/online demos/corina_demo Sybyl 8.0 (Tripos). Accessed 29 Dec 2019

Côté S, Carmichael PH, Verreault R, Lindsay J, Lefebvre J, Laurin D (2012) Nonsteroidal anti-inflammatory drug use and the risk of cognitive impairment and Alzheimer's disease. Alzheimers Dement. 8(3):219-226. https://doi.org/10.1016/j.jalz.2011.03.012

Cudaback E, Jorstad NL, Yang Y, Montine TJ, Keene CD (2014) Therapeutic implications of the prostaglandin pathway in Alzheimer's disease. Biochem Pharmacol 88(4):565-572. https://doi. org/10.1016/j.bcp.2013.12.014

Czarnecka K, Szymański P, Girek M, Mikiciuk-Olasik E, Skibiński R, Kabziński J, Majsterek I, Malawska B, Jończyk J, Bajda M (2017) Tetrahydroacridine derivatives with fluorobenzoic acid moiety as multifunctional agents for Alzheimer's disease treatment. Bioorg Chem 72:315-322. https://doi.org/10.1016/j.bioorg.2017.05.003

Czarnecka K, Chufarova N, Halczuk K, Maciejewska K, Girek M, Skibiński R, Jończyk J, Bajda M, Kabziński J, Majsterek I, 
Szymański P (2018) Tetrahydroacridine derivatives with dichloronicotinic acid moiety as attractive, multipotent agents for Alzheimer's disease treatment. Eur J Med Chem 145:760-769. https ://doi.org/10.1016/j.ejmech.2018.01.014

Ellman GL, Courtney KD, Andres V, Featherstone RM (1961) A new and rapid colorimetric determination of acetylcholinesterase activity. Biochem Pharmacol 7(2):88-95. https://doi.org/10.1016/00062952(61)90145-9

Fernandez-Bachiller MI, Perez C, Gonzalez-Munoz GC, Conde S, Lopez MG, Villarroya M, Garcia AG, Rodriguez-Franco MI (2010) Novel tacrine-8-hydroxyquinoline hybrids as multifunctional agents for the treatment of alzheimer's disease, with neuroprotective, cholinergic, antioxidant, and copper-complexing properties. J Med Chem 53(13):4927-4937. https://doi.org/10.1021/ jm $100329 \mathrm{q}$

Gasteiger J, Marsili M (1978) A new model for calculating atomic charges in molecules. Tetrahedron Lett 19(34):3181-3184. https ://doi.org/10.1016/S0040-4039(01)94977-9

Huang L, Miao H, Sun Y, Meng F, Li X (2014) Discovery of indanone derivatives as multi-target-directed ligands against Alzheimer's disease. Eur J Med Chem 87:429-439. https://doi.org/10.1016/j. ejmech.2014.09.081

Imbimbo BP, Solfrizzi V, Panza F (2010) Are NSAIDs useful to treat alzheimer's disease or mild cognitive impairment? Front Aging Neurosci 2:19. https://doi.org/10.3389/fnagi.2010.00019

Kurz A, Perneczky R (2011) Novel insights for the treatment of Alzheimer's disease. Prog Neuropsychopharmacol Biol Psychiatry 35(2):373-379. https://doi.org/10.1016/j.pnpbp.2010.07.018

Li SY, Wang XB, Xie SS, Jiang N, Wang KDG, Yao HQ, Sun HB, Kong LY (2013) Multifunctional tacrine-flavonoid hybrids with cholinergic, $\beta$-amyloid-reducing, and metal chelating properties for the treatment of Alzheimer's disease. Eur J Med Chem 69:632-646. https://doi.org/10.1016/j.ejmech.2013.09.024

Lu H, Li X, Zhang J, Shi HUI, Zhu X, He X (2014) Effects of cordycepin on HepG2 and EA.hy926 cells: Potential antiproliferative, antimetastatic and anti-angiogenic effects on hepatocellular carcinoma. Oncol Lett 7(5):1556-1562. https://doi.org/10.3892/ ol.2014.1965

Mailloux A, Grenet K, Bruneel A, Bénéteau-Burnat B, Vaubourdolle M, Baudin B (2001) Anticancer drugs induce necrosis of human endothelial cells involving both oncosis and apoptosis. Eur J Cell Biol 80(6):442-449. https://doi.org/10.1078/0171-9335-00171

McGeer PL (2000) Cyclo-oxygenase-2 inhibitors. Drugs Aging 17(1):1-11. https://doi.org/10.2165/00002512-200017010-00001

Medina M, Avila J (2014) New perspectives on the role of tau in Alzheimer's disease. Implications for therapy. Biochem Pharmacol 88(4):540-547. https://doi.org/10.1016/j.bcp.2014.01.013

Meraz-Ríos MA, Toral-Rios D, Franco-Bocanegra D, Villeda-Hernández J, Campos-Peña V (2013) Inflammatory process in Alzheimer's disease. Front Integr Neurosci 7:59. https://doi.org/10.3389/ fnint.2013.00059

Musil K, Florianova V, Bucek P, Dohnal V, Kuca K, Musilek K (2016) Development and validation of a FIA/UV-vis method for $\mathrm{pKa}$ determination of oxime based acetylcholinesterase reactivators. J Pharm Biomed Anal 117:240-246. https://doi.org/10.1016/j. jpba.2015.09.010

Nenadis N, Lazaridou O, Tsimidou MZ (2007) Use of reference compounds in antioxidant activity assessment. J Agric Food Chem 55(14):5452-5460. https://doi.org/10.1021/jf070473q

Ng YP, Or TCT, Ip NY (2015) Plant alkaloids as drug leads for Alzheimer's disease. Neurochem Int 89:260-270. https://doi. org/10.1016/j.neuint.2015.07.018

Obulesu M, Jhansilakshmi M (2014) Neuroinflammation in Alzheimer's disease: an understanding of physiology and pathology. Int J Neurosci 124(4):227-235. https://doi.org/10.3109/00207 454.2013 .831852
Park SM, Ki SH, Han NR, Cho IJ, Ku SK, Kim SC, Zhao RJ, Kim YW (2015) Tacrine, an oral acetylcholinesterase inhibitor, induced hepatic oxidative damage, which was blocked by liquiritigenin through GSK3-beta inhibition. Biol Pharm Bull 38(2):184-192. https://doi.org/10.1248/bpb.b14-00430

Plumb JA (1999) Cell sensitivity assays: the MTT assay. Methods Mol Med 28:25-30. https://doi.org/10.1385/1-59259-687-8:25

Prati F, Bartolini M, Simoni E, De Simone A, Pinto A, Andrisano V, Bolognesi ML (2013) Quinones bearing non-steroidal antiinflammatory fragments as multitarget ligands for Alzheimer's disease. Bioorg Med Chem Lett 23(23):6254-6258. https://doi. org/10.1016/j.bmcl.2013.09.091

PyMOL 0.99rc6 (2006) DeLano Scientific LLC: Palo Alto, CA, USA

Re R, Pellegrini N, Proteggente A, Pannala A, Yang M, Rice-Evans C (1999) Antioxidant activity applying an improved ABTS radical cation decolorization assay. Free Radic Biol Med 26(9):12311237. https://doi.org/10.1016/S0891-5849(98)00315-3

Results of a follow-up study to the randomized Alzheimer's Disease Anti-inflammatory Prevention Trial (ADAPT) (2013) Alzheimer's \& dementia. J Alzheimer's Assoc 9(6):714-723. https:// doi.org/10.1016/j.jalz.2012.11.012

Solleiro-Villavicencio H, Rivas-Arancibia S (2018) Effect of chronic oxidative stress on neuroinflammatory response mediated by $\mathrm{CD} 4+\mathrm{T}$ cells in neurodegenerative diseases. Front Cell Neurosci 12(114):1-13. https://doi.org/10.3389/fncel.2018.00114

Sybyl-X 1.1 (2010) Tripos, St. Louis, MO, USA

Szymanski P, Karpinski A, Mikiciuk-Olasik E (2011) Synthesis, biological activity and HPLC validation of 1,2,3,4-tetrahydroacridine derivatives as acetylcholinesterase inhibitors. Eur J Med Chem 46(8):3250-3257. https://doi.org/10.1016/j.ejmech.2011.04.038

Tayeb H, Yang H, Price B, Tarazi F (2012) Pharmacotherapies for Alzheimer's disease: beyond cholinesterase inhibitors. Pharmacol Ther 134(1):8-25. https://doi.org/10.1016/j.pharmthera .2011.12.002

Technical Bulletin (2019) CellTiter $96^{\circledR} \mathrm{AQ}_{\text {ueous }}$ One Solution Cell Proliferation Assay. https://pl.promega.com/products/cell-healt h-assays/cell-viability-and-cytotoxicity-assays/celltiter-96-aqueo us-one-solution-cell-proliferation-assay-_mts_/?catNum=G3582 . Accessed 29 Dec 2019

Townsend KP, Praticò D (2005) Novel therapeutic opportunities for Alzheimer's disease: focus on nonsteroidal anti-inflammatory drugs. FASEB J 19(12):1592-1601. https://doi.org/10.1096/ fj.04-3620rev

Volpato D, Holzgrabe U (2018) Designing hybrids targeting the cholinergic system by modulating the muscarinic and nicotinic receptors: a concept to treat alzheimer's disease. Molecules 23:3230. https://doi.org/10.3390/molecules23123230

USP XXII-NF (1990) XVII United States Pharmacopeia Convention, Inc., pp 644-645

Zemek F, Drtinova L, Nepovimova E, Sepsova V, Korabecny J, Klimes J, Kuca K (2014) Outcomes of Alzheimer's disease therapy with acetylcholinesterase inhibitors and memantine. Expert Opin Drug Saf 13(6):759-774. https://doi.org/10.1517/14740338.2014.91416 8

Zimmer ER, Leuzy A, Benedet AL, Breitner J, Gauthier S, Rosa-Neto $P$ (2014) Tracking neuroinflammation in Alzheimer's disease: the role of positron emission tomography imaging. J Neuroinflammation 11:120. https://doi.org/10.1186/1742-2094-11-120

Publisher's Note Springer Nature remains neutral with regard to jurisdictional claims in published maps and institutional affiliations. 